\title{
Faithful actions from hyperplane arrangements
}

\author{
YUKI HIRANO \\ Michael WeMYSS
}

\begin{abstract}
We show that if $X$ is a smooth quasiprojective 3-fold admitting a flopping contraction, then the fundamental group of an associated simplicial hyperplane arrangement acts faithfully on the derived category of $X$. The main technical advance is to use torsion pairs as an efficient mechanism to track various objects under iterations of the flop functor (or mutation functor). This allows us to relate compositions of the flop functor (or mutation functor) to the theory of Deligne normal form, and to give a criterion for when a finite composition of 3-fold flops can be understood as a tilt at a single torsion pair. We also use this technique to give a simplified proof of a result of Brav and Thomas (Math. Ann. 351 (2011) 1005-1017) for Kleinian singularities.
\end{abstract}

18E30; 14E30, 14F05, 14J30, 20F36

\section{Introduction}

Autoequivalence groups of the bounded derived categories $\mathrm{D}^{\mathrm{b}}(\operatorname{coh} X)$ of coherent sheaves of varieties $X$ have been studied in many articles. On one hand, Bondal and Orlov [2] proved that derived categories $\mathrm{D}^{\mathrm{b}}(\operatorname{coh} X)$ of smooth projective varieties $X$ with $K_{X}$ or $-K_{X}$ ample have only standard autoequivalences. On the other hand, Seidel and Thomas [26] showed that if $\pi: X \rightarrow \mathbb{C}^{2} / G$ is a minimal resolution of a quotient singularity $\mathbb{C}^{2} / G$ by a finite group $G \subset \mathrm{SL}_{2}(\mathbb{C})$, then the derived category $\mathrm{D}^{\mathrm{b}}(\operatorname{coh} X)$ has nonstandard autoequivalences, called spherical twists. Across mirror symmetry, these correspond to autoequivalences of the derived Fukaya category of a homological mirror partner $X^{\vee}$ of $X$, which arises from generalised Dehn twists along Lagrangian spheres in $X^{\vee}$ [26]. More precisely, if $C:=\pi^{-1}(0)=\bigcup_{i=1}^{n} C_{i}$ with $C_{i}$ irreducible, Seidel and Thomas showed that the objects $\mathcal{O}_{C_{i}}(-1)[1]$ induce autoequivalences $t_{i} \in$ Auteq $\mathrm{D}^{\mathrm{b}}(\operatorname{coh} X)$, and that these together induce a group homomorphism

$$
\rho: B_{\Gamma} \rightarrow \text { Auteq }^{\mathrm{b}}(\operatorname{coh} X), \quad s_{i} \mapsto t_{i},
$$

where $B_{\Gamma}=\left\langle s_{1}, \ldots, s_{n}\right\rangle$ is the braid group of the dual graph of exceptional curves $\bigcup_{i=1}^{n} C_{i}$ of $\pi$, which is a Dynkin diagram of type ADE. Seidel and Thomas showed 
that $\rho$ is injective when $\Gamma$ is of type $A$, and later Brav and Thomas [3] showed that $\rho$ is injective in the general case. This means that there is a faithful braid group action on $\mathrm{D}^{\mathrm{b}}(\operatorname{coh} X)$.

Moving up one dimension, if $X \rightarrow X_{\text {con }}$ is a flopping contraction between quasiprojective 3 -folds, where $X$ is smooth and each of the $n$ irreducible exceptional curves is individually floppable, then Pinkham [23] and Wemyss [29] associate to this data a real hyperplane arrangement $\mathcal{H} \subseteq \mathbb{R}^{n}$, as a certain intersection in an ADE root system. The main result of Donovan and Wemyss [10] is that this induces an action of the fundamental group on the derived category; more precisely, there exists a group homomorphism

$$
\varphi: \pi_{1}\left(\mathbb{C}^{n} \backslash \mathcal{H}_{\mathbb{C}}\right) \rightarrow \text { Auteq } \mathrm{D}^{\mathrm{b}}(\operatorname{coh} X),
$$

where $\mathcal{H}_{\mathbb{C}}$ denotes the complexification of the real hyperplane arrangement $\mathcal{H} \subseteq \mathbb{R}^{n}$. The group $\pi_{1}\left(\mathbb{C}^{n} \backslash \mathcal{H}_{\mathbb{C}}\right)$ should be viewed as a form of pure braid group, since in the case that $\mathcal{H}$ is a Coxeter arrangement, this is precisely what it is. However, in general, $\mathcal{H}$ need not be Coxeter. Motivated by the situation of surfaces above, and also by considerations in Bridgeland stability conditions, we prove that $\varphi$ is injective, that is, the action is also faithful.

In fact, we do more, and our proof also recovers the surfaces case of [3] in a much simpler way. Some of the techniques in [3] are not suited to the 3-fold and more general settings, and so we are forced to develop a new approach. There are four main problems:

(1) In the 3-fold flops setting, the action $\varphi$ is obtained by iterating flops. There is no "formula" for the flop functor, unlike for spherical twists, and so tracking objects under iterated flops is much more challenging.

(2) The arrangement $\mathcal{H}$ need not be Coxeter, so there is no finite Weyl group from which we can use reduced expressions of elements, or Garside normal form.

(3) Higher-length braid relations exist, making it harder to induct on path length.

(4) There is no explicit presentation of $\pi_{1}\left(\mathbb{C}^{n} \backslash \mathcal{H}_{\mathbb{C}}\right)$ to work with.

It turns out that these phenomena also exist for surfaces, but we need to go to partial resolutions of Kleinian singularities in order to see them; most work to date only considers the minimal resolution. This is addressed further in Iyama and Wemyss [16]. To obtain our main geometric results, we restrict to the formal fibre, and manipulate tilting modules there. The following is our main result: 
Theorem 1.1 (Theorem 6.5 and Corollary 6.6) Suppose that $f: X \rightarrow$ Spec $\Re$ is a complete local 3-fold flopping contraction, where $X$ is smooth. Then the natural functor from the Deligne groupoid $\mathbb{G}_{\mathcal{H}}$ to the natural flops groupoid is faithful. In particular, the induced group homomorphism

$$
\varphi: \pi_{1}\left(\mathbb{C}^{n} \backslash \mathcal{H}_{\mathbb{C}}\right) \rightarrow \text { Auteq } \mathrm{D}^{\mathrm{b}}(\operatorname{coh} X)
$$

is injective.

This immediately gives global corollaries, such as the following:

Corollary 6.7 Suppose that $f: X \rightarrow X_{\text {con }}$ is a flopping contraction between quasiprojective 3-folds, where $X$ is smooth, and all curves in the contraction $f$ are individually floppable. Then there is an injective group homomorphism

$$
\varphi: \pi_{1}\left(\mathbb{C}^{n} \backslash \mathcal{H}_{\mathbb{C}}\right) \rightarrow \text { Auteq } \mathrm{D}^{\mathrm{b}}(\operatorname{coh} X) .
$$

There is a similar statement for when the curves are not individually floppable, but being slightly more technical to state, we refer the reader to Corollary 6.8. We also recover in Appendix A a simplified version of Brav and Thomas in the case of minimal resolutions of Kleinian singularities.

The main technical engine in the proof is to use the order on tilting modules to control iterations. Our new main technical result is the following, which here we state slightly vaguely, leaving details to Section 4.

Theorem 1.2 (Theorem 4.6) With the assumptions in Theorem 1.1, suppose that $\alpha: C \rightarrow D$ is a positive minimal path. Then the composition of mutation functors along this path is functorially isomorphic to a single functor induced by a tilting module.

Since tilting modules induce torsion pairs, this allows us to use torsion pairs to control iterations. Applying this to 3-fold flops, where by Wemyss [29] the flop functor is isomorphic to the inverse of the mutation functor, gives the following result. The first part is implicit in Donovan and Wemyss [10], whereas the second part is new, and may be of independent interest.

Theorem 6.9 Consider two crepant resolutions

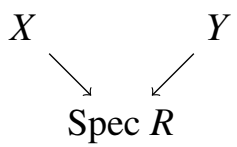

of Spec $R$, where $R$ is an isolated $c D V$ singularity. 
(1) Given two minimal chains of flops connecting $X$ and $Y$, the composition of flop functors associated to each chain are functorially isomorphic.

(2) Perverse sheaves on $Y$, namely ${ }^{0} \operatorname{Per}(Y, R)$, can be obtained from perverse sheaves on $X$, namely ${ }^{0} \operatorname{Per}(X, R)$, by a single tilt at a torsion pair.

For definitions, we refer the reader to Section 6.2.

\subsection{Outline of the paper}

Section 2 contains background on hyperplane arrangements, arrangement groupoids and Deligne normal form. In Section 3 we then relate this to tilting modules, under the general setting that we will consider. So as not to disturb the flow of the paper, proofs of some of the results in Section 3 appear in Appendix B. In Section 4 we establish in Theorem 4.6 that compositions of tilts behave well under Deligne normal form, and the first consequences appear in the short Section 5. In Section 6 we use this torsion pair viewpoint to prove the faithfulness in the complete local setting, and we give all the geometric corollaries. In Appendix A, which can be read independently, we give a simple direct proof of faithfulness in the case of Kleinian singularities, to demonstrate that the torsion pair viewpoint simplifies the proof.

\subsection{Conventions}

All rings and algebras are assumed to be noetherian, and to be $k$-algebras, where $k$ is some field. All modules are right modules, unless stated otherwise. When considering flopping contractions, the base field is assumed to be algebraically closed of characteristic zero. Throughout:

- For a triangulated category $\mathcal{C}$, and $a, b \in \mathcal{C}$, to match [3] we write

$$
[a, b]_{t}:=\operatorname{Hom}_{\mathcal{C}}(a, b[t]) .
$$

- For an algebra $\Lambda$, we write $\mathrm{fl} \Lambda$ for the category of finite-length right $\Lambda$-modules.

- For a noetherian ring $R, \mathrm{CM} R$ denotes the category of maximal CohenMacaulay $R$-modules, and ref $R$ denotes the category of finitely generated reflexive $R$-modules.

- For an additive category $\mathcal{C}$ and an object $x \in \mathcal{C}$, we write add $x \subset \mathcal{C}$ for the full subcategory consisting of direct summands of finite direct sums of $x$. 


\section{Acknowledgements}

Wemyss would like to thank Osamu Iyama for discussions related to the tilting theory in Appendix B. Both authors would like to thank the referee for their patience, and for their helpful comments. The majority of this work was carried out when Hirano visited the Universities of Edinburgh and Glasgow during 2015/16, funded by the JSPS. We thank the JSPS, and also the universities for their hospitality.

Hirano was a Research Fellow for the Japan Society for the Promotion of Science, and was partially supported by Grant-in-Aid 26-6240. Wemyss was supported by EPSRC grant EP/K021400/2.

\section{Preliminaries}

\subsection{Hyperplane arrangements}

Throughout this subsection $\mathcal{H}$ will denote a finite set of hyperplanes in $\mathbb{R}^{n}$, which we will refer to as a real hyperplane arrangement. Such an arrangement is called Coxeter if it arises as the set of reflection hyperplanes of a finite real reflection group.

Recall that $\mathcal{H}$ is simplicial if $\bigcap_{H \in \mathcal{H}} H=\{0\}$ and all chambers in $\mathbb{R}^{n} \backslash \mathcal{H}$ are open simplicial cones. All Coxeter arrangements are simplicial, but the converse is false. When $\mathcal{H}$ is simplicial, we will write

$$
\mathcal{H}_{\mathbb{C}}:=\bigcup_{H \in \mathcal{H}} H_{\mathbb{C}}
$$

where $H_{\mathbb{C}}$ denotes the complexification of $H$. The fundamental object of interest to us is the fundamental group $\pi_{1}\left(\mathbb{C}^{n} \backslash \mathcal{H}_{\mathbb{C}}\right)$ and, as is standard, to access this combinatorially we will use the Deligne groupoid in the next subsection.

Remark 2.1 When $\mathcal{H}$ is Coxeter, it is well known that $\pi_{1}\left(\mathbb{C}^{n} \backslash \mathcal{H}_{\mathbb{C}}\right)$ is the pure braid group associated to the corresponding finite Coxeter group, that is, the kernel of the natural morphism from the braid group to the Weyl group. When the arrangement is simplicial but not Coxeter, there is no such description in terms of a kernel.

When $\mathcal{H}$ is a simplicial hyperplane arrangement, its 1 -skeleton is defined to be the graph with vertices corresponding to the chambers, and edges joining chambers which share a codimension-one wall. 
Example 2.2 As an example, consider the following hyperplane arrangement $\mathcal{H}$ in $\mathbb{R}^{3}$, and its 1-skeleton. It has 7 hyperplanes and 32 chambers, and is not Coxeter:
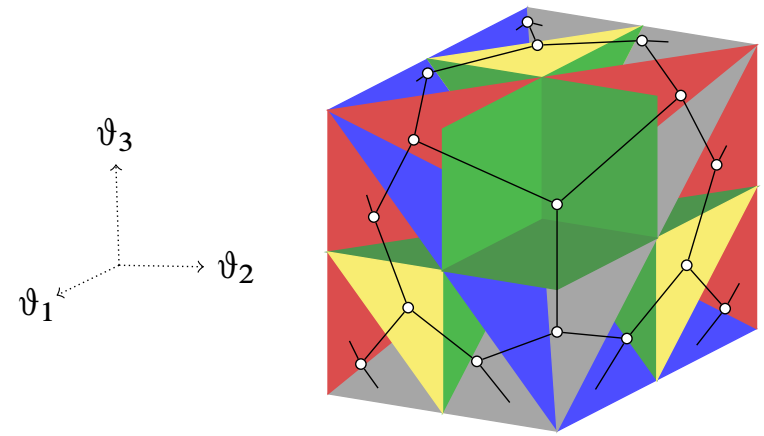

$$
\begin{aligned}
\vartheta_{1} & =0, \\
\vartheta_{2} & =0, \\
\vartheta_{3} & =0, \\
\vartheta_{1}+\vartheta_{2} & =0, \\
\vartheta_{1}+\vartheta_{3} & =0, \\
\vartheta_{2}+\vartheta_{3} & =0, \\
\vartheta_{1}+\vartheta_{2}+\vartheta_{3} & =0 .
\end{aligned}
$$

This hyperplane arrangement appears for $\mathrm{cD}_{4}$ singularities with three curves meeting at a point $[29,7.4]$; an explicit example of such a $\mathrm{cD}_{4}$ singularity can be found in $[6,11.2 .19]$.

\subsection{The Deligne groupoid}

In this section we summarise some known combinatorial approaches to $\pi_{1}\left(\mathbb{C}^{n} \backslash \mathcal{H}_{\mathbb{C}}\right)$. For more detailed references, see $[21 ; 22 ; 7]$.

Recall that a groupoid is a small category $\mathcal{G}$ such that for any two objects $g, h \in \mathcal{G}$, the set of morphisms $\operatorname{Hom}(g, h)$ is nonempty and, further, all morphisms are invertible. We recall that a hyperplane arrangement $\mathcal{H}$ in $\mathbb{R}^{n}$ induces a groupoid $\mathbb{G}_{\mathcal{H}}$ called the arrangement groupoid (or Deligne groupoid) of $\mathcal{H}$. To define this, we first associate an oriented graph $\Gamma_{\mathcal{H}}$ to the hyperplane arrangement $\mathcal{H}$.

Definition 2.3 The vertices of $\Gamma_{\mathcal{H}}$ are the chambers (ie the connected components) of $\mathbb{R}^{n} \backslash \bigcup_{H \in \mathcal{H}} H$. There is an arrow $a: v_{1} \rightarrow v_{2}$ from chamber $v_{1}$ to chamber $v_{2}$ if the chambers are adjacent, otherwise there is no arrow. For an arrow $a: v_{1} \rightarrow v_{2}$, we set $s(a):=v_{1}$ and $t(a):=v_{2}$.

Example 2.4 Consider the hyperplane arrangement $\mathcal{H}$ in $\mathbb{R}^{2}$, and its associated $\Gamma_{\mathcal{H}}$ in Figure 1. We have labelled the arrows in $\Gamma_{\mathcal{H}}$ by abuse of notation.

A positive path of length $n$ in $\Gamma_{\mathcal{H}}$ is defined to be a formal symbol

$$
p=a_{n} \circ \cdots \circ a_{2} \circ a_{1}
$$



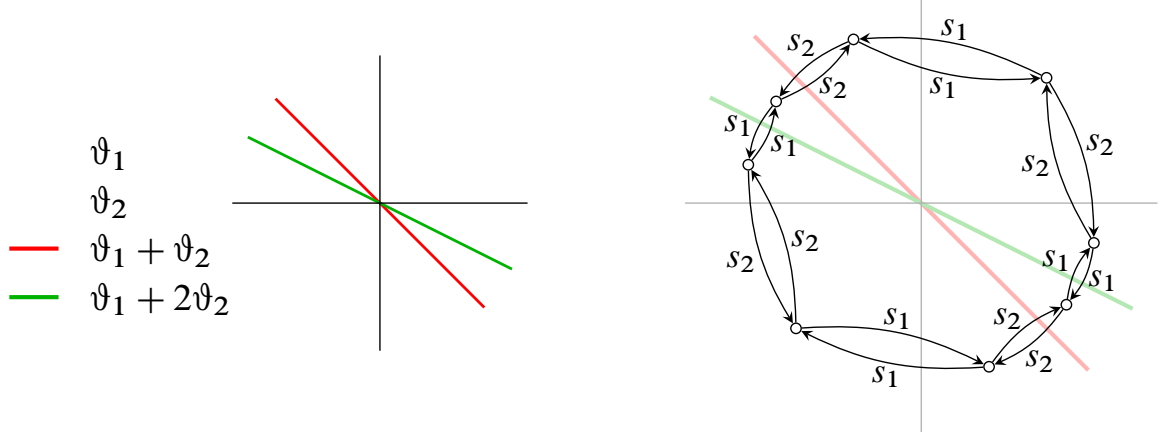

Figure 1

whenever there exists a sequence of vertices $v_{0}, \ldots, v_{n}$ of $\Gamma_{\mathcal{H}}$ and arrows $a_{i}: v_{i-1} \rightarrow v_{i}$ in $\Gamma_{\mathcal{H}}$. We define $s(p):=v_{0}, t(p):=v_{n}$ and $\ell(p):=n$. The notation $\circ$ should remind us of composition, but we will often drop the $\circ$ in future sections. If $q=b_{m} \circ \cdots \circ b_{2} \circ b_{1}$ is another positive path with $t(p)=s(q)$, we consider the formal symbol

$$
q \circ p:=b_{m} \circ \cdots \circ b_{2} \circ b_{1} \circ a_{n} \circ \cdots \circ a_{2} \circ a_{1},
$$

and call it the composition of $p$ and $q$. As usual, there are paths of length zero at each vertex $v$, and by abuse of notation we will also denote the length-zero path at $v$ by $v$, and identify the compositions $t(p) \circ p$ and $p \circ s(p)$ with $p$.

Definition 2.5 A positive path is called minimal if there is no positive path in $\Gamma_{\mathcal{H}}$ of smaller length and with the same endpoints. The positive minimal paths are called atoms.

Example 2.6 In Example 2.4, the following are all the atoms starting in the chamber $C_{+}$:

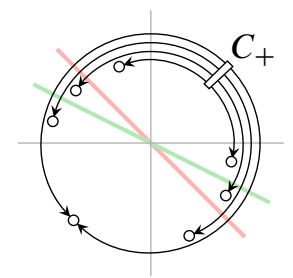

For each choice of start chamber, there is a similar picture.

Following [21, page 170], there is an equivalence relation $\sim$ on the set of paths in $\Gamma_{\mathcal{H}}$, defined as the smallest equivalence relation such that the following conditions are satisfied: 
(1) If $p \sim q$, then $s(p)=s(q)$ and $t(p)=t(q)$.

(2) If $p$ and $q$ are atoms with the same source and targets, then $p \sim q$.

(3) If $p \sim q$, then $u p r \sim u q r$ for all positive paths $u$ and $r$ satisfying $t(r)=$ $s(p)=s(q)$ and $s(u)=t(p)=t(q)$.

Write Path $\Gamma_{\mathcal{H}}$ for the set of equivalence classes of positive paths in $\Gamma_{\mathcal{H}}$ with respect to the equivalence relation $\sim$, and write $[p]$ for the equivalence class of a positive path $p$.

Definition 2.7 When $\mathcal{H}$ is a simplicial hyperplane arrangement, write $\mathbb{G}_{\mathcal{H}}^{+}$for the category whose objects are the vertices in $\Gamma_{\mathcal{H}}$, and whose morphisms are defined

$$
\operatorname{Hom}_{\mathbb{G}_{\mathcal{H}}^{+}}(v, u):=\left\{[p] \in \operatorname{Path} \Gamma_{\mathcal{H}} \mid s(p)=v \text { and } t(p)=u\right\} .
$$

The Deligne groupoid (or the arrangement groupoid) $\mathbb{G}_{\mathcal{H}}$ is the groupoid defined as the groupoid completion of $\mathbb{G}_{\mathcal{H}}^{+}$, that is, adding formal inverses of all morphisms in $\mathbb{G}_{\mathcal{H}}^{+}$ (see eg [9, Section 2.3.1]).

In future sections, we will abuse notation, and refer to $[\alpha] \in$ Path $\Gamma_{\mathcal{H}}$ simply by $\alpha$, with the equivalence relation being implicit. The following is well known by $[7 ; 21 ; 20 ; 25]$ (see also [22, 2.1]), and is our main reason for considering the Deligne groupoid.

Theorem 2.8 If $\mathcal{H}$ is simplicial, any vertex group of the groupoid $\mathbb{G}_{\mathcal{H}}$ defined above is isomorphic to $\pi_{1}\left(\mathbb{C}^{n} \backslash \mathcal{H}_{\mathbb{C}}\right)$.

\subsection{Faithfulness}

The faithfulness of the action of $\pi_{1}\left(\mathbb{C}^{n} \backslash \mathcal{H}_{\mathbb{C}}\right)$ on $\mathrm{D}^{\mathrm{b}}(\operatorname{coh} X)$ will follow from a more general faithful result on groupoids, which we briefly outline here.

Definition 2.9 [7, Section 1] Assume that $\mathcal{H}$ is simplicial. Let $v_{i}$ and $v_{j}$ be vertices in $\Gamma_{\mathcal{H}}$, and let $C_{i}$ and $C_{j}$ be the corresponding chambers of $\mathbb{R}^{n} \backslash \bigcup_{H \in \mathcal{H}} H$. Then we say that $v_{j}$ is opposite to $v_{i}$ if there is a line $l$ in $\mathbb{R}^{n}$ passing through $C_{i}, C_{j}$ and the origin. An opposite vertex of $v$ is unique, and we denote it by $-v$.

Lemma 2.10 Assume that $\mathcal{H}$ is simplicial.

(1) For any atom $p$ in $\Gamma_{\mathcal{H}}$, there is an atom $p^{\prime}$ such that $s\left(p^{\prime}\right)=-t(p), t\left(p^{\prime}\right)=$ $s(p)$ and the composition $p p^{\prime}$ is also an atom. 
(2) Let $a$ and $b$ be two atoms in $\Gamma_{\mathcal{H}}$ such that $t(a)=t(b)$. Then there are atoms $p$ and $q$ such that $b^{-1} a=q p^{-1}$ in $\operatorname{Hom}_{\mathbb{G}_{\mathcal{H}}}(s(a), s(b))$.

Proof (1) This follows from [21, Section 4, Corollary 2].

(2) By (1), there are atoms $p$ and $q$ such that $s(p)=s(q)=-t(a)$, and $a p$ and $b q$ are atoms. Since the targets and sources of $a p$ and $b q$ are equal, we have $a p \sim b q$. This implies $b^{-1} \circ a=q \circ p^{-1}$ in $\operatorname{Hom}_{\mathbb{G}_{\mathcal{H}}}(s(a), s(b))$.

Since $\mathbb{G}_{\mathcal{H}}$ is obtained from $\mathbb{G}_{\mathcal{H}}^{+}$by adding inverses, there is a natural functor

$$
\iota: \mathbb{G}_{\mathcal{H}}^{+} \rightarrow \mathbb{G}_{\mathcal{H}} .
$$

The following lemma is an easy analogue of [3, Lemma 2.3], and relies on the fact that $\iota$ is faithful for simplicial $\mathcal{H}$.

Lemma 2.11 Assume that $\mathcal{H}$ is simplicial, and let $F: \mathbb{G}_{\mathcal{H}} \rightarrow \mathcal{G}$ be a functor between groupoids. Then $F$ is faithful if and only if $F \circ \iota: \mathbb{G}_{\mathcal{H}}^{+} \rightarrow \mathcal{G}$ is faithful.

Proof By [7], ı: $\mathbb{G}_{\mathcal{H}}^{+} \rightarrow \mathbb{G}_{\mathcal{H}}$ is faithful. Thus, it immediately follows that if $F$ is faithful, so is $F \circ \iota: \mathbb{G}_{\mathcal{H}}^{+} \rightarrow \mathcal{G}$.

For the other direction, assume that $F \circ \iota: \mathbb{G}_{\mathcal{H}}^{+} \rightarrow \mathcal{G}$ is faithful and let $p, q \in \operatorname{Hom}_{\mathbb{G}_{\mathcal{H}}}(v, w)$ be morphisms. It is enough to show that if $F(p)=F(q)$ then $p=q$. At first, we consider the case when $v=w$. In this case, it is enough to show that, if $F(p)=\mathrm{id}_{F(v)}$, then $p=\mathrm{id}_{v}$. By repeated use of Lemma 2.10(2), there are positive paths $p_{1}$ and $p_{2}$ such that $p=p_{1} \circ p_{2}{ }^{-1}$. Since we have $F\left(p_{1}\right)=F\left(p_{2}\right)$ and $F \circ \iota$ is faithful, necessarily $p_{1}=p_{2}$ and so $p=p_{1} \circ p_{2}{ }^{-1}=\mathrm{id}_{v}$. Next, we consider the general case when $F(p)=F(q)$. Then we have $F\left(p q^{-1}\right)=\mathrm{id}_{F\left(v_{j}\right)}$. By the above argument, we see that $p q^{-1}=\mathrm{id}_{v}$, and thus $p=q$.

Corollary 2.12 Assume that $\mathcal{H}$ is simplicial, $F: \mathbb{G}_{\mathcal{H}} \rightarrow \mathcal{G}$ is a functor between groupoids and, for any chamber $C$, write $\operatorname{Autgp}(F C):=\operatorname{Hom}_{\mathcal{G}}(F C, F C)$. If $F$ is a faithful functor, then there is an injective group homomorphism

$$
\pi_{1}\left(\mathbb{C}^{n} \backslash \mathcal{H}_{\mathbb{C}}\right) \rightarrow \operatorname{Autgp}(F C) .
$$

Proof If $F$ is faithful, the induced group homomorphism $F: \operatorname{Hom}_{\mathbb{G}_{\mathcal{H}}}(C, C) \rightarrow$ $\operatorname{Autgp}(F C)$ is injective for any chamber $C \in \mathbb{G}_{\mathcal{H}}$. Since $\operatorname{Hom}_{\mathbb{G}_{\mathcal{H}}}(C, C)$ is isomorphic to $\pi_{1}\left(\mathbb{C}^{n} \backslash \mathcal{H}_{\mathbb{C}}\right)$ by Theorem 2.8 , the result holds. 


\subsection{Deligne normal form}

By Lemma 2.11 and Corollary 2.12, our problem will reduce to proving the faithfulness of a positive part of a groupoid action. This is a significant reduction in complexity, since every positive path has a Deligne normal form, which we recall here. This normal form replaces the Garside normal form in [3], which is only defined for Coxeter arrangements. The proof of faithfulness will simply induct on the number of factors of this normal form.

For positive paths $p, q \in \Gamma_{\mathcal{H}}$ with $s(p)=s(q)$, we say that $p$ begins with $q$ if there exists a positive path $r$ such that $s(r)=t(q), t(r)=t(p)$ and $p \sim r q$. For a positive path $p$, write $\operatorname{Begin}(p)$ for the set of all atoms with which $p$ begins. Similarly, we can consider the set of atoms with which $p$ ends, which is defined in the analogous way, and we denote this set by $\operatorname{End}(p)$.

Definition 2.13 For any path $p \in \Gamma_{\mathcal{H}}$, by $[22,2.2]$ (or [7]), there exists a unique (up to equivalence) atom $\alpha_{1}$ such that $\operatorname{Begin}(p)=\operatorname{Begin}\left(\alpha_{1}\right)$. Then, in particular, $p$ begins with $\alpha_{1}$, and so there is a positive path $\beta$ with $s(\beta)=t\left(\alpha_{1}\right)$ and $t(\beta)=t(p)$ such that

$$
p \sim \beta \circ \alpha_{1}
$$

Continuing this process with $\beta$, we decompose $p$ into atoms

$$
p \sim \alpha_{n} \circ \cdots \circ \alpha_{2} \circ \alpha_{1},
$$

which we refer to as the Deligne normal form of $p$.

The following lemma is convenient, and is well known [21, Lemma 4.2].

Lemma 2.14 If $p \in \Gamma_{\mathcal{H}}$, then $p$ is an atom if and only if $p$ does not cross any hyperplane twice.

Example 2.15 Continuing the example and notation in Example 2.4, dropping the composition symbol $\circ$, the path $p=s_{2} s_{1} s_{2} s_{1} s_{2} s_{2} s_{1} s_{1} s_{2} s_{1}$ satisfies $\operatorname{Begin}(p)=$ $\operatorname{Begin}\left(s_{2} s_{1} s_{2} s_{1}\right)$ since

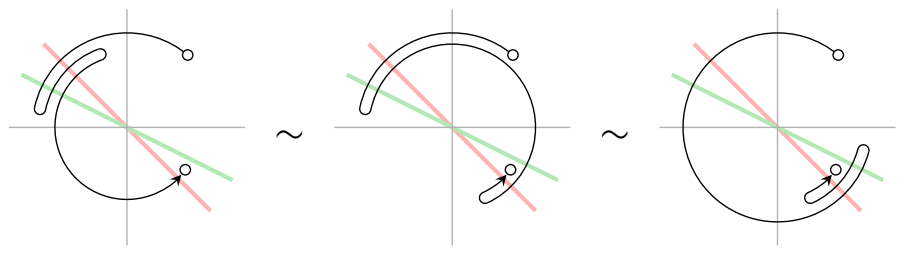

Continuing in this way, $p$ has Deligne normal form $s_{2}\left(s_{2} s_{1}\right)\left(s_{1} s_{2} s_{1}\right)\left(s_{2} s_{1} s_{2} s_{1}\right)$. 


\section{The tilting order and chambers}

Our strategy to prove faithfulness of the action in the flops setting is to exploit the partial order on tilting modules, due to Riedtmann and Schofield [24] and Happel and Unger [13]. In the case of minimal resolutions of Kleinian singularities, we can bypass this step by simply appealing to [15, Section 6], and so for the proof of faithfulness in this case, the reader can skip immediately to Appendix A.

\subsection{Tilting modules and mutation}

Recall first that for an algebra $A$ such that the category $\bmod A$ of finitely generated $A$-modules is Krull-Schmidt, $M \in \bmod A$ is called basic if there is no repetition in its Krull-Schmidt decomposition into indecomposable $A$-modules, and the algebra $A$ is called basic if it is basic as an $A$-module.

Throughout this section, $\Lambda$ is a basic $\mathfrak{R}$-algebra, where $\mathfrak{R}$ is a complete local domain. Note, by [28, page 566], for such rings the category $\bmod \Lambda$ is Krull-Schmidt. In our geometric settings later, such $\Lambda$ appear when we work on the formal fibre.

Definition 3.1 $T \in \bmod \Lambda$ is a classical tilting module if the following conditions hold:

(1) $\operatorname{pd}_{\Lambda} T \leq 1$.

(2) $\operatorname{Ext}_{\Lambda}^{1}(T, T)=0$.

(3) There exists a short exact sequence $0 \rightarrow \Lambda \rightarrow T_{1} \rightarrow T_{2} \rightarrow 0$ with each $T_{i} \in$ add $T$.

We write tilt $\Lambda$ for the set of basic classical tilting $\Lambda$-modules.

We shall refer to classical tilting modules simply as tilting modules, with it being implicit that $\operatorname{pd}_{\Lambda} T \leq 1$. When $T$ is a tilting module, we write Fac $T$ for the full subcategory of $\bmod \Lambda$ consisting of those modules $Y$ such that there exists a surjection $T^{\prime} \rightarrow Y$ with $T^{\prime} \in$ add $T$. It is known, and easy to prove from Definition 3.1(3), that

$$
\operatorname{Fac} T=\left\{X \in \bmod \Lambda \mid \operatorname{Ext}_{\Lambda}^{1}(T, X)=0\right\},
$$

so in particular for any $X \in$ Fac $T$ there is an exact sequence

$$
0 \rightarrow Y \rightarrow T^{\prime} \rightarrow X \rightarrow 0
$$


with $Y \in \operatorname{Fac} T$ and $T^{\prime} \in$ add $T$. It follows immediately that

$$
\operatorname{add} T=\left\{X \in \operatorname{Fac} T \mid \operatorname{Ext}_{\Lambda}^{1}(X, \operatorname{Fac} T)=0\right\} .
$$

The set tilt $\Lambda$ carries the natural structure of a partially ordered set.

Notation 3.2 Let $T, U \in$ tilt $\Lambda$. We write $T \geq U$ if $\operatorname{Ext}_{\Lambda}^{1}(T, U)=0$, or, equivalently by (3.A), if $U \in \operatorname{Fac} T$. We write $T>U$ if $T \geq U$ and $\neg(U \geq T)$.

It is immediate from (3.B) and the Krull-Schmidt property that if $T, U \in$ tilt $\Lambda$ with $T \geq U \geq T$, then $T \cong U$. We remark that $T \geq U$ if and only if Fac $T \supseteq$ Fac $U$, and that $\Lambda \in$ tilt $\Lambda$ is the greatest element with respect to $\geq$.

Another key property of the set tilt $\Lambda$ is that it admits an operation called mutation. For $T \in$ tilt $\Lambda$ and an indecomposable direct summand $T_{i}$ of $T$, there exists at most one basic tilting $\Lambda$-module $\nu_{i} T=\left(T / T_{i}\right) \oplus U_{i}$ such that $T_{i} \not U_{i}$ (cf [24]). The module $\nu_{i} T$ is called a tilting mutation of $T$, and in general it may or may not exist. As is standard, mutation is encoded in the exchange graph of tilt $\Lambda$.

Notation 3.3 We write $\mathrm{EG}(\Lambda)$ for the exchange graph, where vertices are elements of tilt $\Lambda$, and we draw an edge between $T$ and $\nu_{i} T$ for all $T$ and $i$ such that $\nu_{i} T$ exists. Further, for a fixed projective $P$, let $\mathrm{EG}_{P}(\Lambda)$ denote the full subgraph of the exchange graph of $\Lambda$ consisting of those vertices that contain $P$ as a summand.

\subsection{Chambers associated to tilting modules}

To functorially control compositions of tilting mutations requires chambers, which we now describe. We first fix notation. Let $\Lambda$ be a basic $\mathfrak{R}$-algebra, where $\mathfrak{R}$ is a complete local domain, and write $K_{0}:=K_{0}\left(\mathrm{~K}^{\mathrm{b}}(\operatorname{proj} \Lambda)\right)$. It is well known that

$$
K_{0} \cong \mathbb{Z}^{n+1}
$$

since every $P \in \operatorname{proj} \Lambda$ can be uniquely written as a direct sum of indecomposable projectives $P_{0}^{\oplus a_{0}} \oplus \cdots \oplus P_{n}^{\oplus a_{n}}$ for some $a_{i}$. In what follows, we will fix the $\mathbb{Z}$-basis of $K_{0}$ given by (3.C), namely $\left\{\boldsymbol{e}_{0}, \ldots, \boldsymbol{e}_{n}\right\}$, where $\boldsymbol{e}_{i}$ is the class of $P_{i}$ in $K_{0}$.

We now fix a projective, which by convention will be $P_{0}$, and we will primarily be interested in $\mathrm{EG}_{0}(\Lambda):=\mathrm{EG}_{P_{0}}(\Lambda)$, and its vertex set tilt $\mathrm{t}_{0}(\Lambda)$ consisting of all tilting $\Lambda$-modules that contain $P_{0}$ as a summand. For this purpose, consider the following factor $\mathbb{R}$-vector space of $K_{0} \otimes_{\mathbb{Z}} \mathbb{R} \cong \mathbb{R}^{n+1}$, given by

$$
\Theta_{\Lambda}:=\left(K_{0} \otimes_{\mathbb{Z}} \mathbb{R}\right) / \operatorname{Span}\left\{\boldsymbol{e}_{0}\right\} \cong \mathbb{R}^{n} .
$$


By abuse of notation, we write $\left\{\left[P_{1}\right], \ldots,\left[P_{n}\right]\right\}$ for the $\mathbb{R}$-basis of $\Theta_{\Lambda}$ induced by (3.C), with it being implicit that the [-] notation works modulo $\operatorname{Span}\left\{\boldsymbol{e}_{0}\right\}$. From this, we define

$$
C_{+}:=\left\{\sum_{i=1}^{n} \vartheta_{i}\left[P_{i}\right] \mid \vartheta_{i}>0 \text { for all } 1 \leq i \leq n\right\} \subseteq \Theta_{\Lambda} .
$$

For $T \in$ tilt $_{0} \Lambda$, write $T=T_{0} \oplus T_{1} \oplus \cdots \oplus T_{n}$, where by convention $P_{0}=T_{0}$, and consider

$$
C_{T}:=\left\{\sum_{i=1}^{n} \vartheta_{i}\left[T_{i}\right] \mid \vartheta_{i}>0 \text { for all } 1 \leq i \leq n\right\} \subseteq \Theta_{\Lambda} .
$$

It is clear from the definition that $C_{\Lambda}=C_{+}$.

The following is elementary, and is very similar to the arguments of [14;8]. Since the setting here does not involve Hom-finite categories, we give the proof in Appendix B.

Lemma 3.4 Suppose that $\Lambda$ is a basic $\mathfrak{R}$-algebra, where $\mathfrak{R}$ is a complete local domain. If $T, U \in$ tilt $_{0} \Lambda$ are related by a mutation at an indecomposable summand, then $C_{T}$ and $C_{U}$ do not overlap, and are separated by a codimension-one wall.

It is the following that will allow us to control iterations, as it relates the combinatorics of chamber structures to the homological property of the tilting order. The result seems to be folklore; for lack of a suitable reference, and since we are working slightly more generally than usual, we give the proof in Theorem B.4 in Appendix B.

Theorem 3.5 Suppose that $\Lambda$ is a basic $\mathfrak{R}$-algebra, where $\mathfrak{R}$ is a complete local domain. Suppose that $T, U \in$ tilt $_{0} \Lambda$ are related by a mutation at an indecomposable summand, so, by Lemma 3.4, $C_{T}$ and $C_{U}$ are separated by $H$. Suppose that $[\Lambda] \notin H$. Then $T>U$ if and only if $C_{T}$ lies on the same side of $H$ as $[\Lambda]$.

\section{Compositions of mutations and flops}

In this section we will describe compositions of mutation functors, or flop functors, under Deligne normal form. This, and more generally the proof of faithfulness of the group action, will be reduced to the formal fibre, and so for much of the paper we will work under the following setup:

Setup 4.1 Suppose that $f: U \rightarrow$ Spec $\mathfrak{R}$ is a complete local 3-fold flopping contraction, where $U$ is smooth. 
It is well known $[1,3.2 .8]$ that in this setting $\mathrm{D}^{\mathrm{b}}(\operatorname{coh} U)$ admits a tilting bundle $\mathcal{V}$ generated by global sections, which after setting $M:=f_{*} \mathcal{V}$, induces an equivalence $\mathrm{D}^{\mathrm{b}}(\operatorname{coh} U) \cong \mathrm{D}^{\mathrm{b}}\left(\bmod \operatorname{End}_{\mathfrak{R}}(M)\right)$. The algebra $\operatorname{End}_{\mathfrak{R}}(M)$ contains $\operatorname{Hom}_{\mathfrak{R}}(M, \mathfrak{R})$ as a summand, and in the following we fix $P_{0}:=\operatorname{Hom}_{\mathfrak{R}}(M, \mathfrak{R})$, so that tilt ${ }_{0}(\Lambda)$ consists of those tilting $\Lambda$-modules containing $\operatorname{Hom}_{\mathfrak{R}}(M, \mathfrak{R})$ as a summand.

\subsection{CT objects and simple wall crossings}

Under the above flops setup, $M \in \mathrm{CM} \Re$ and $\operatorname{End}_{\mathfrak{R}}(M)$ is a NCCR [1, 3.2.9-3.2.10]. It follows $[17,5.4]$ that $M$ is a cluster tilting (CT) object of CM $\Re$, namely there are equalities

$$
\text { add } M=\left\{X \in \mathrm{CM} \Re \mid \operatorname{Ext}_{\mathfrak{R}}^{1}(X, M)=0\right\}=\left\{Y \in \mathrm{CM} \mathfrak{R} \mid \operatorname{Ext}_{\mathfrak{R}}^{1}(M, Y)=0\right\} .
$$

We can, and will, assume that $M$ is basic. The class of basic cluster tilting objects carries an operation of mutation, which involves picking an indecomposable summand $M_{i}$ of a CT module $M$, and uniquely replacing it with a different indecomposable summand whilst remaining CT; the resulting module will be denoted by $\nu_{i} M$.

By the three-dimensional Auslander-McKay correspondence [29, 6.9], the number of CT $\mathfrak{R}$-modules is equal to the number of chambers of some simplicial hyperplane arrangement, described in detail in [29, 5.24-5.25], and furthermore, crossing a codimension-one wall (henceforth a simple wall crossing) corresponds to mutating an indecomposable summand of the associated CT module. Consequently, the 1-skeleton of the arrangement equals the exchange graph of CT $\mathfrak{R}$-modules.

Under Setup 4.1, to fix notation we will write $\mathcal{H}_{\Lambda}$ for the simplicial hyperplane arrangement associated to $f$, set $M:=f_{*} \mathcal{V}$, which will correspond to the chamber $C_{+}$, and fix $\Lambda:=\operatorname{End}_{\Re}(M)$.

Example 4.2 There exists [19] a $\mathrm{cD}_{4}$ flop with the following simplicial hyperplane arrangement. Under the Auslander-McKay correspondence, Figure 2 illustrates the exchange graph of CT objects, where $v_{i_{2} i_{1}}:=v_{i_{2}} v_{i_{1}}$ etc.

Thus, under Setup 4.1, via [29, 5.24-5.25] every chamber $C$ in $\mathcal{H}_{\Lambda}$ has an associated CT $\mathfrak{R}$-module, $N_{C}$ say, and thus an associated derived category $\mathrm{D}^{\mathrm{b}}\left(\bmod \Lambda_{C}\right)$, where $\Lambda_{C}:=\operatorname{End}_{\Re}\left(N_{C}\right)$. The following Notation 4.3 shows that there are natural equivalences between the categories. 


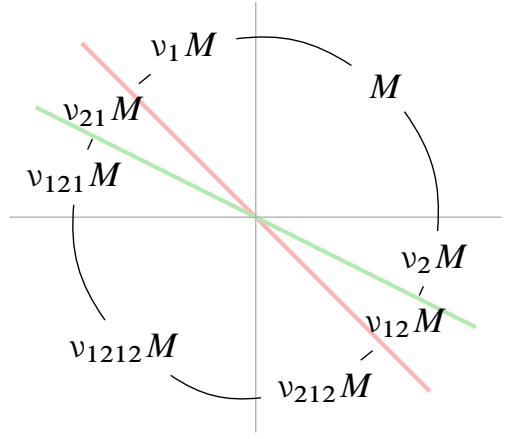

Figure 2

Notation 4.3 Suppose that $\alpha: C \rightarrow D$ is an atom in $\Gamma_{\mathcal{H}_{\Lambda}}$. Then, by [17, 4.17], $T_{C D}:=\operatorname{Hom}_{\Re}\left(N_{C}, N_{D}\right)$ is a tilting bimodule from $\Lambda_{C}$ to $\Lambda_{D}$, and we consider the equivalence

$$
\mathrm{D}^{\mathrm{b}}\left(\bmod \Lambda_{C}\right) \stackrel{t_{\alpha}:=\operatorname{RHom}_{\Lambda_{C}}\left(T_{C D},-\right)}{\longrightarrow} \mathrm{D}^{\mathrm{b}}\left(\bmod \Lambda_{D}\right)
$$

When $\alpha$ is a simple wall crossing, mutating the $i^{\text {th }}$ summand of $N_{C}$ say, we will write

$$
t_{i}:=\mathbf{R H o m}_{\Lambda_{C}}\left(T_{C D},-\right),
$$

and refer to $t_{i}$ as the mutation functor.

Remark 4.4 By [29, Theorem 4.2], the functor $t_{i}$ is functorially isomorphic to the inverse of the flop functor, flopping a single curve $C_{i}$.

It is known [10, 3.22] that the mutation functors $t_{i}$ form a representation of the Deligne groupoid, and thus they alone are enough to induce the action of the fundamental group. However, it is the existence of the additional functors $t_{\alpha}$ for every atom $\alpha$ that will allow us to control this action, and prove faithfulness in this paper.

Example 4.5 Continuing Example 4.2, setting $\Lambda_{I}:=\operatorname{End}_{\mathfrak{R}}\left(\nu_{I} M\right)$, the mutation functors $t_{i}$ are as in Figure 3.

There are more direct functors, for all atoms. As in Example 2.6, for those out of $C_{+}$ these are as in Figure 4.

There are similar additional functors emerging from each of the other chambers. 


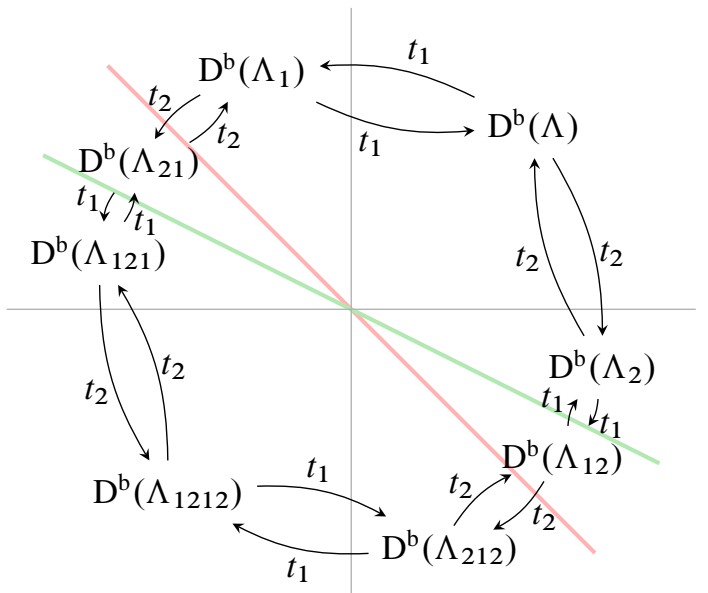

Figure 3

\subsection{Atoms and the tilting order}

Under the flops setup, Setup 4.1, recall from the last subsection that we associate an algebra $\Lambda=\operatorname{End}_{\mathfrak{R}}(M)$, and a simplicial hyperplane arrangement $\mathcal{H}_{\Lambda}$. The functor

$$
\mathbb{F}:=\operatorname{Hom}_{\mathfrak{R}}(M,-): \bmod R \rightarrow \bmod \Lambda
$$

is fully faithful, and furthermore, by $[17,4.17,5.11]$, induces an injective map

$\mathbb{F}:\{\mathrm{CT} \Re$-modules $\} \rightarrow$ tilt $_{0} \Lambda$,

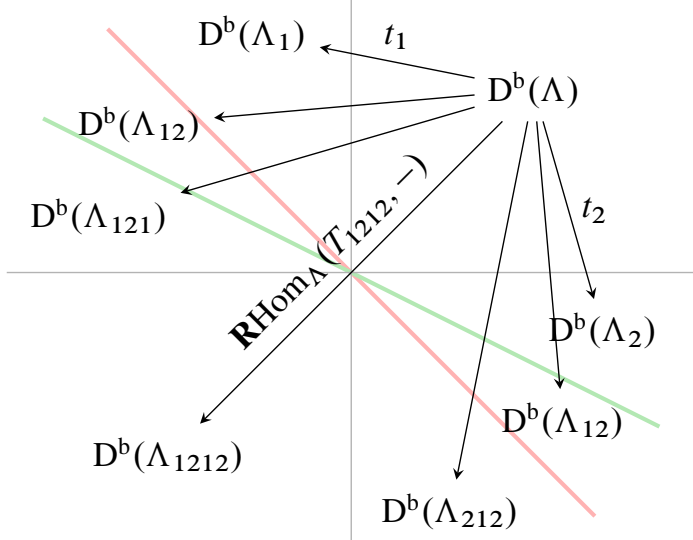

Figure 4 
where recall tilt 0 consists of all tilting $\Lambda$-modules containing $P_{0}=\operatorname{Hom}_{\mathfrak{R}}(M, \mathfrak{R})$ as a summand. By $[18,4.5(1)]$ this map is compatible with mutation. But, since $\mathfrak{R}$ is an isolated singularity, all possible mutations of a fixed CT $\mathfrak{R}$-module $N$ give all possible mutations of $\mathbb{F} N$ in tilt ${ }_{0} \Lambda$, hence the finite connected mutation graph of CT $\mathfrak{R}$-modules induces, under $\mathbb{F}$, a finite connected component of tilt $t_{0} \Lambda$. By a result of Happel and Unger (adapted and proved in the setting here in $[18,4.9]$ ), tilt ${ }_{0} \Lambda$ must equal this finite connected component, thus (4.A) is in fact a bijection compatible with mutation.

It follows that the exchange graph $\mathrm{EG}_{0} \Lambda$ from Section 3.2 equals the exchange graph of CT $\mathfrak{R}$-modules, in a way compatible with mutation. Hence, by the last subsection, $E_{0} \Lambda$ also equals the 1 -skeleton of $\mathcal{H}_{\Lambda}$, and thus the chambers of $\mathcal{H}_{\Lambda}$ are indexed by tilting $\Lambda$-modules, in a manner such that two modules that share a codimensionone wall are related by a mutation at an indecomposable summand, in the sense of Section 3.1. We refer the reader to Example 4.8 for an example.

The following is our main technical lemma, which uses the tilting chambers to establish in the second part that the composition of mutation functors along Deligne normal form is given by a direct tilt. To avoid confusion, write $D_{T}$ for the chamber of $\mathcal{H}_{\Lambda}$ indexed by $T \in$ tilt $_{0} \Lambda$, and write $C_{T}$ for the chamber (3.D). We write $D_{+}:=D_{\Lambda}$.

Theorem 4.6 Under Setup 4.1, for any $S \in$ tilt $_{0} \Lambda$, suppose that $\alpha: D_{+} \rightarrow D_{S}$ is an atom in $\Gamma_{\mathcal{H}_{\Lambda}}$, and choose a decomposition of $\alpha$ into length-one positive paths

$$
\alpha=D_{\Lambda} \stackrel{s_{i_{1}}}{\longrightarrow} D_{2} \rightarrow \cdots \rightarrow D_{m} \stackrel{s_{i m}}{\longrightarrow} D_{m+1} .
$$

For $i=2, \ldots, m+1$, write $M_{i}$ for the $C T \mathfrak{R}$-module corresponding to the chamber $D_{i}$, so that $S=\mathbb{F} M_{m+1}$. Then the following assertions hold:

(1) As tilting $\Lambda$-modules, $\Lambda=\mathbb{F} M>\mathbb{F} M_{2}>\cdots>\mathbb{F} M_{m}>\mathbb{F} M_{m+1}=S$.

(2) There is a bimodule isomorphism

$$
\begin{aligned}
& \operatorname{Hom}_{\mathfrak{R}}\left(M_{m}, M_{m+1}\right) \otimes^{\boldsymbol{L}} \cdots \otimes^{\boldsymbol{L}} \operatorname{Hom}_{\mathfrak{R}}\left(M_{2}, M_{3}\right) \otimes^{\boldsymbol{L}} \operatorname{Hom}_{\mathfrak{R}}\left(M, M_{2}\right) \\
& \cong \operatorname{Hom}_{\mathfrak{R}}\left(M, M_{m+1}\right),
\end{aligned}
$$

where, reading right to left, the tensors are over $\operatorname{End}_{\mathfrak{R}}\left(M_{i}\right)$ for $i=2, \ldots, m$.

(3) $C_{S}:=\left\{\sum_{i=1}^{n} \vartheta_{i}\left[S_{i}\right] \mid \vartheta_{i}>0\right.$ for all $\left.1 \leq i \leq n\right\}$ equals $D_{S}$.

Proof We prove all assertions together. By induction we can assume that

$$
\Lambda=\mathbb{F} M>\cdots>\mathbb{F} M_{m},
$$


that $C_{X}=D_{X}$ for the tilting modules in (4.B), and that there is a bimodule isomorphism

$$
\operatorname{Hom}_{\mathfrak{R}}\left(M_{m-1}, M_{m}\right) \otimes^{L} \cdots \otimes^{L} \operatorname{Hom}_{\mathfrak{R}}\left(M, M_{2}\right) \cong \operatorname{Hom}_{\mathfrak{R}}\left(M, M_{m}\right),
$$

since the case $m=1$ is clear.

Certainly the hyperplanes of $\mathcal{H}_{\Lambda}$ cannot pass through any chamber, in particular $C_{\Lambda}=D_{\Lambda}$. Write $T:=\mathbb{F} M_{m}$, and $H^{\prime}$ for the wall separating $C_{T}$ and $C_{S}$. Since $C_{T}=D_{T}$ by induction, extending $H^{\prime}$ to a hyperplane, $H^{\prime}$ is one of the hyperplanes of $\mathcal{H}_{\Lambda}$. Hence, since $[\Lambda] \in C_{\Lambda}$, and the hyperplanes of $\mathcal{H}_{\Lambda}$ cannot pass through $C_{\Lambda}$, necessarily $[\Lambda] \notin H^{\prime}$.

We next crash through the wall $H$ from $D_{T}$ into $D_{S}$. If $D_{+}$were not on the same side of $H$ as $D_{T}$, then $\alpha$ would have to cross $H$ twice, and so by Lemma 2.14 applied to $\mathcal{H}_{\Lambda}$, the path $\alpha$ would not be an atom. Hence, $D_{+}$must be on the same side of $H$ as $D_{T}$. Since $[\Lambda] \in C_{+}=D_{+}$and $C_{T}=D_{T}$, we conclude that $[\Lambda]$ is on the same side of $H^{\prime}$ as $C_{T}$.

By Theorem 3.5, necessarily $T>S$, ie $\mathbb{F} M_{m}>\mathbb{F} M_{m+1}$, so combining with (4.B) proves (1). Next, the induction (4.C) gives a bimodule isomorphism

$\operatorname{Hom}_{\Re}\left(M_{m}, M_{m+1}\right) \otimes^{L}\left(\cdots \otimes \otimes^{L} \operatorname{Hom}_{\Re}\left(M, M_{2}\right)\right)$

$$
\cong \operatorname{Hom}_{\mathfrak{R}}\left(M_{m}, M_{m+1}\right) \otimes^{L} \operatorname{Hom}_{\mathfrak{R}}\left(M, M_{m}\right),
$$

so to prove (2) it suffices to show that there is a bimodule isomorphism

$$
\operatorname{Hom}_{\mathfrak{R}}\left(M_{m}, M_{m+1}\right) \otimes^{L} \operatorname{Hom}_{\mathfrak{R}}\left(M, M_{m}\right) \cong \operatorname{Hom}_{\mathfrak{R}}\left(M, M_{m+1}\right) .
$$

Applying Proposition B.1 with $T=\mathbb{F} M_{m}, \Gamma=\operatorname{End}_{\Re}\left(M_{m}\right)$ and

$$
\nu_{i} \Gamma=\operatorname{Hom}_{\mathfrak{R}}\left(M_{m}, M_{m+1}\right)
$$

shows that the left-hand side of (4.D) is concentrated in degree zero, so to prove (2) it suffices to show that there is a bimodule isomorphism

$$
\operatorname{Hom}_{\mathfrak{R}}\left(M_{m}, M_{m+1}\right) \otimes \operatorname{Hom}_{\mathfrak{R}}\left(M, M_{m}\right) \cong \operatorname{Hom}_{\mathfrak{R}}\left(M, M_{m+1}\right) .
$$

But there is a chain of isomorphisms

$$
\operatorname{Hom}_{\mathfrak{R}}\left(M_{m}, M_{m+1}\right) \otimes \operatorname{Hom}_{\mathfrak{R}}\left(M, M_{m}\right) \stackrel{\sim}{\longrightarrow} \operatorname{Hom}_{\Lambda}\left(T, \mathbb{F} M_{m+1}\right) \otimes T \stackrel{\sim}{\longrightarrow} M_{m+1},
$$

where the first is the reflexive equivalence $g \otimes f \mapsto(g \circ-) \otimes f$, and the second is the adjunction from the derived equivalence (using the last statement in Proposition B.1), which takes $\varphi \otimes t \mapsto \varphi(t)$. Composing the above shows that there is an isomorphism 
(4.E) given by $g \otimes f \mapsto g \circ f$. By inspection, this an isomorphism in the category of bimodules, proving (2).

Finally, to prove (3), note that the bimodule isomorphism in (2) induces a functorial isomorphism between $\mathbf{R H o m}_{\Lambda}\left(\mathbb{F} M_{m+1},-\right)=\operatorname{RHom}_{\Lambda}(S,-)$ and the composition

$$
\mathrm{D}^{\mathrm{b}}(\bmod \Lambda) \stackrel{t_{i_{1}}}{\longrightarrow} \cdots \stackrel{t_{i_{m}}}{\longrightarrow} \mathrm{D}^{\mathrm{b}}\left(\bmod \Lambda_{m+1}\right) \text {. }
$$

Writing $\Lambda_{m+1}=P_{0} \oplus Q_{1} \oplus \cdots \oplus Q_{n}$, it is easy to see that tracking

$$
\left\{\sum_{i} \vartheta_{i}\left[Q_{i}\right] \mid \vartheta_{i}>0, i=1, \ldots, n\right\}
$$

through the inverse of $\operatorname{RHom}_{\Lambda}(S,-)$ gives

$$
\left\{\sum_{i} \vartheta_{i}\left[S_{i}\right] \mid \vartheta_{i}>0, i=1, \ldots, n\right\}=C_{S}
$$

By the functorial isomorphism, this must give the same answer as tracking (4.G) through the inverse of (4.F). We thus claim that tracking (4.G) through the inverse of (4.F) gives $D_{S}$, as then $D_{S}=C_{S}$, and the result follows.

On one hand, by the definition of the mutation functors, tracking (4.G) through the inverse of (4.F) precisely follows the moduli-tracking rules laid out in [29, 5.14-5.15]. On the other hand, it is known $[29,5.25]$ that after possibly replacing some of the

$$
t_{j}=\mathbf{R H o m}_{\operatorname{End}_{\mathfrak{R}}(N)}\left(\operatorname{Hom}_{\mathfrak{R}}\left(N, \nu_{j} N\right),-\right)
$$

in (4.F) by

$$
t_{j}^{\prime}:=-\otimes_{\operatorname{End}_{\mathfrak{R}}(N)}^{\boldsymbol{L}} \operatorname{Hom}_{\mathfrak{R}}\left(v_{j} N, N\right),
$$

tracking (4.G) back through the inverse of the replacement chain does indeed give the simplicial cone $D_{S}$. Crucially, since the combinatorial rules for tracking through $t_{j}$ and through $t_{j}^{\prime}$ are the same in this flops setting (see $[29,5.15]$ ), the replacements do not matter, and so tracking (4.G) through the inverse of (4.F) also gives $D_{S}$, as required.

Remark 4.7 The initial choice of decomposition of $\alpha$ in Theorem 4.6 does not matter, as the theorem shows that all choices are functorially isomorphic to $t_{\alpha}$.

Example 4.8 Continuing the flopping contraction example in Example 4.5, the chambers of $\mathcal{H}_{\Lambda}$ can be indexed by elements of tilt $t_{0} \Lambda$, as illustrated in the left-hand side of the following picture, where $\nu_{i_{2} i_{1}} \Lambda=\operatorname{Hom}_{\mathfrak{R}}\left(M, \nu_{i_{2} i_{1}} M\right)$ etc: 


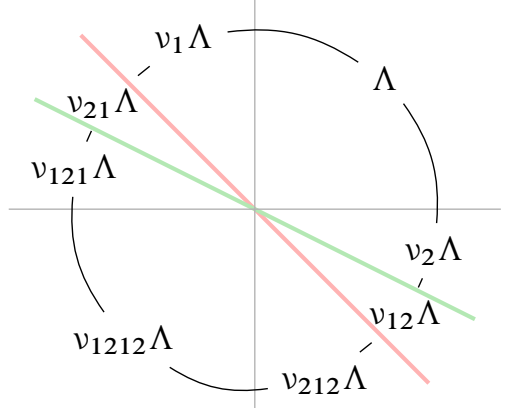

$\mathrm{EG}_{0}(\Lambda)$

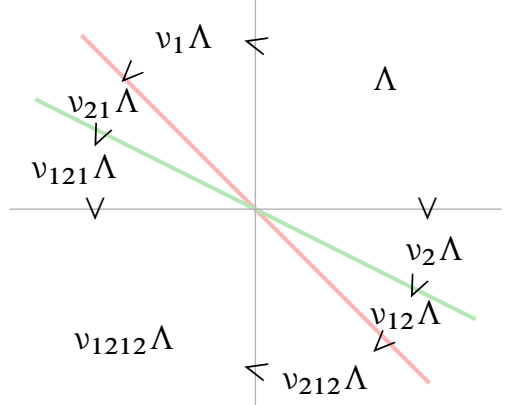

Order

The ordering, which is illustrated in the right-hand side, is forced by Theorem 4.6(1).

Since the positive path below corresponding to the composition $t_{2} t_{1} t_{2}$ is an atom, it also follows from Theorem 4.6(2) (applied to $\Lambda_{1}$ ) that the composition $t_{2} t_{1} t_{2}$ is functorially isomorphic to the direct functor shown:

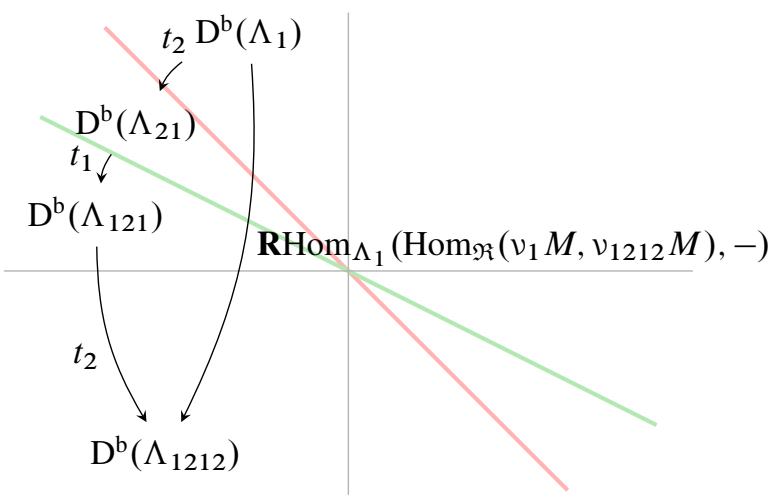

For future use, a useful corollary of Theorem 4.6 is the following:

Corollary 4.9 Under Setup 4.1, let $\alpha: C \rightarrow D$ be an atom, and let $N \in \bmod \Lambda_{C}$. Then $H^{i}\left(t_{\alpha} N\right)=0$ for all $i \neq 0,1$.

\section{Tracking via torsion pairs}

Under the flops setup of Setup 4.1, suppose that $C$ is a chamber of $\mathcal{H}_{\Lambda}$. It follows from Theorem 4.6 applied to $\Lambda_{C}=\operatorname{End}_{\mathfrak{R}}\left(N_{C}\right)$ that if $\alpha: C \rightarrow D$ is an atom, then the composition of mutation functors along the path $\alpha$ is functorially isomorphic to 
$t_{\alpha}=\operatorname{RHom}_{\Lambda_{C}}\left(T_{\alpha},-\right)$, where $T_{\alpha}:=v_{\alpha} \Lambda_{C} \in$ tilt $_{0} \Lambda_{C}$. We will use this implicitly from now on.

As is standard (see eg [27, 2.7]), $T_{\alpha}$ induces two torsion pairs, which restrict to torsion pairs on finite-length modules $\mathrm{fl} \Lambda_{C}$ and $\mathrm{fl} \Lambda_{D}$. These are $\left(\mathcal{T}_{\alpha}, \mathcal{F}_{\alpha}\right)$ and $\left(\mathcal{X}_{\alpha}, \mathcal{Y}_{\alpha}\right)$, where

$$
\begin{aligned}
& \mathcal{T}_{\alpha}:=\left\{N \in \mathrm{fl} \Lambda_{C} \mid \operatorname{Ext}_{\Lambda_{C}}^{1}\left(T_{\alpha}, N\right)=0\right\}, \\
& \mathcal{F}_{\alpha}:=\left\{N \in \mathrm{fl} \Lambda_{C} \mid \operatorname{Hom}_{\Lambda_{C}}\left(T_{\alpha}, N\right)=0\right\},
\end{aligned}
$$

and

$$
\begin{aligned}
& \mathcal{X}_{\alpha}:=\left\{N \in \text { fl } \Lambda_{D} \mid N \otimes_{\Lambda_{D}} T_{\alpha}=0\right\}, \\
& \mathcal{Y}_{\alpha}:=\left\{N \in \mathrm{fl} \Lambda_{D} \mid \operatorname{Tor}_{1}^{\Lambda_{D}}\left(N, T_{\alpha}\right)=0\right\} .
\end{aligned}
$$

The Brenner-Butler theorem for finite-dimensional algebras (proved in the modulefinite setting here in $[27,2.9]$ ) asserts that these tilting modules not only induce the above two torsion pairs, but also induce the following categorical equivalences:

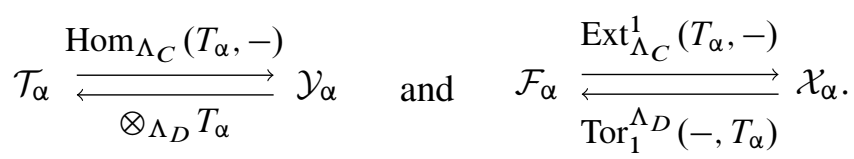

To control the functors $t_{\alpha}$ requires us to track various objects, which we do here. The following lemma is a standard fact about Deligne normal form, which precisely mirrors the Coxeter version.

Lemma 5.1 Suppose that $\alpha$ is an atom. Then:

(1) $\alpha s_{i}$ is an atom $\Longleftrightarrow s_{i} \notin \operatorname{Begin}(\alpha)$.

(2) $s_{j} \alpha$ is an atom $\Longleftrightarrow s_{j} \notin \operatorname{End}(\alpha)$.

Proof For lack of a suitable reference, we give the proof of (1), with (2) being similar. $(\Longrightarrow)$ This is clear, using Lemma 2.14.

$(\Longleftarrow)$ We prove the contrapositive. Suppose that the composition

$$
A \stackrel{s_{i}}{\longrightarrow} B \stackrel{\alpha}{\longrightarrow} C
$$

is not an atom, and write $H$ for the hyperplane separating $A$ and $B$. By Lemma 2.14, $\alpha s_{i}$ must cross some hyperplane at least twice. But, since $\alpha$ is an atom, again by Lemma 2.14, the hyperplanes that $\alpha$ crosses must be distinct. Hence, the only possibility is that $\alpha s_{i}$ crosses $H$ precisely twice. 
In particular, $\alpha$ must cross $H$, and so, since it cannot cross $H$ twice by Lemma 2.14, $t(\alpha)=C$ must be on the same side of $H$ as $A$. If we write $\beta$ for the smallest positive path (atom) from $A$ to $C$, then $\beta$ cannot cross $H$ by Lemma 2.14, since $A$ and $C$ lie on the same side of $H$. Since $s_{i}: B \rightarrow A$ obviously only crosses $H$, it follows again by Lemma 2.14 that the composition

$$
B \stackrel{s_{i}}{\longrightarrow} A \stackrel{\beta}{\longrightarrow} C
$$

is an atom. Hence, $\alpha \sim \beta s_{i}$, since both are atoms from $B$ to $C$, and so $s_{i} \in \operatorname{Begin}(\alpha)$.

Notation 5.2 In each chamber $D$ of $\mathrm{EG}_{0}(\Lambda)$ there is an algebra $\Lambda_{D}$ with precisely $n+1$ simples. By abuse of notation we will denote these simples by $S_{0}, S_{1}, \ldots, S_{n}$, where $S_{0}$ always corresponds to $P_{0}$, and performing the simple wall crossing $s_{i}$ corresponds to the tilting mutation at the projective cover of $S_{i}$. We will use the same notation $S_{i}$ for every $\Lambda_{D}$, and will often consider $\mathcal{S}:=\bigoplus_{i=0}^{n} S_{i}$, with it being implicit from the context which $\Lambda_{D}$ to view this as a module over.

Lemma 5.3 Under Setup 4.1, $t_{i}\left(S_{i}\right) \cong S_{i}[-1]$ for all $1 \leq i \leq n$.

Proof Say $s_{i}: C \rightarrow D$, so that $t_{i}=\mathbf{R H o m}_{\Lambda_{C}}\left(v_{i} \Lambda_{C},-\right)$. Since $\Lambda_{C}>v_{i} \Lambda_{C}$, as in Appendix B there exists a short exact sequence

$$
0 \rightarrow P_{i} \rightarrow P^{\prime} \rightarrow C_{i} \rightarrow 0
$$

with $P^{\prime} \in \operatorname{add}\left(\Lambda_{C} / P_{i}\right)$ such that $v_{i} \Lambda_{C}=\left(\Lambda_{C} / P_{i}\right) \oplus C_{i}$. Applying $\operatorname{Hom}_{\Lambda_{C}}\left(-, S_{i}\right)$ to the above sequence yields the result, exactly as in $[29,4.15(2)]$.

For our purposes later, we require more than Lemma 5.3, namely for atoms $\alpha: C \rightarrow D$ we need to track all summands of $\mathcal{S}$ under the inverse functor $t_{\alpha}^{-1} \cong-\otimes_{\Lambda_{D}}^{L} T_{\alpha}$. Since $\left(\mathcal{X}_{\alpha}, \mathcal{Y}_{\alpha}\right)$ is a torsion pair on fl $\Lambda_{D}$, and each $S_{i}$ is simple, either $S_{i} \in \mathcal{X}_{\alpha}$ or $S_{i} \in \mathcal{Y}_{\alpha}$. Using the categorical equivalences (5.A) it thus follows that

$$
t_{\alpha}^{-1}\left(S_{i}\right)= \begin{cases}\operatorname{Tor}_{1}^{\Lambda_{D}}\left(S_{i}, T_{\alpha}\right)[1] & \text { if } S_{i} \in \mathcal{X}_{\alpha}, \\ S_{i} \otimes_{\Lambda_{D}} T_{\alpha} & \text { if } S_{i} \in \mathcal{Y}_{\alpha} .\end{cases}
$$

In the top case, $t_{\alpha}^{-1}\left(S_{i}\right)$ is the shift of a module in $\mathcal{F}_{\alpha}$, and in the bottom case, $t_{\alpha}^{-1}\left(S_{i}\right)$ is a module in $\mathcal{T}_{\alpha}$.

The following is our key preparatory lemma, which says that the torsion pairs $\left(\mathcal{T}_{\alpha}, \mathcal{F}_{\alpha}\right)$ and $\left(\mathcal{X}_{\alpha}, \mathcal{Y}_{\alpha}\right)$ detect both how $\alpha$ starts and how $\alpha$ ends. 
Lemma 5.4 Under Setup 4.1, suppose that $\alpha: C \rightarrow D$ is an atom. Then, for $i \neq 0$, the following statements hold:

(1) $S_{i} \in \mathcal{F}_{\alpha} \Longleftrightarrow s_{i} \in \operatorname{Begin}(\alpha)$.

(2) $S_{i} \in \mathcal{T}_{\alpha} \Longleftrightarrow s_{i} \notin \operatorname{Begin}(\alpha)$.

(3) $S_{i} \in \mathcal{X}_{\alpha} \Longleftrightarrow s_{i} \in \operatorname{End}(\alpha)$.

(4) $S_{i} \in \mathcal{Y}_{\alpha} \Longleftrightarrow s_{i} \notin \operatorname{End}(\alpha)$.

Proof We prove (1), with all others being similar.

$(\Longleftarrow)$ Suppose that $\alpha$ starts with $s_{i}$, and write $\alpha=\beta \circ s_{i}$. Then $t_{\alpha}\left(S_{i}\right)=t_{\beta}\left(S_{i}\right)[-1]$ by Lemma 5.3. Applying Corollary 4.9 to both sides, it follows that $H^{j}\left(t_{\alpha}\left(S_{i}\right)\right)=0$ for all $j \neq 1$, so $S_{i} \in \mathcal{F}_{\alpha}$.

$(\Longrightarrow)$ Suppose that $\alpha$ does not start with $s_{i}$; then, by Lemma 5.1, $\alpha \circ s_{i}$ is still an atom. Hence, $t_{\alpha s_{i}}=t_{\alpha} \circ t_{s_{i}}$, and so $t_{\alpha s_{i}}\left(S_{i}\right)=t_{\alpha}\left(S_{i}\right)[-1]$ by Lemma 5.3. Thus $t_{\alpha}\left(S_{i}\right)=t_{\alpha s_{i}}\left(S_{i}\right)[1]$, so again applying Corollary 4.9 to both sides, it follows that $H^{j}\left(t_{\alpha}\left(S_{i}\right)\right)=0$ for all $j \neq 0$, so $S_{i} \in \mathcal{T}_{\alpha}$. In particular, $S_{i} \notin \mathcal{F}_{\alpha}$.

Lemma 5.5 Under Setup 4.1, suppose that $\alpha: C \rightarrow D$ is an atom. Then $S_{0} \in \mathcal{T}_{\alpha}$ and $S_{0} \in \mathcal{Y}_{\alpha}$.

Proof The first statement holds since $P_{0}$ is a summand of $T_{\alpha}$, so $\operatorname{Hom}_{\Lambda_{C}}\left(T_{\alpha}, S_{0}\right) \neq 0$. Thus, $S_{0} \notin \mathcal{F}_{\alpha}$ and so, since $S_{0}$ is simple, necessarily $S_{0} \in \mathcal{T}_{\alpha}$.

The second statement is similar, but uses the duality on tilting modules, so we sketch the proof. To ease notation set $A:=\Lambda_{C}, B:=\Lambda_{D}$, and $T:=T_{\alpha}$. By convention the simple right $A$-module $S_{0}$ corresponds to the indecomposable projective $P_{0}$ of $A$, so consider the idempotent $e_{0}$ such that $P_{0}=e_{0} A$. Similarly, $B \cong \operatorname{End}_{A}(T)$ has an idempotent $e_{0}^{\prime}$ corresponding to the summand $e_{0} A$ in the decomposition $T=e_{0} A \oplus X$ as right $A$-modules. By convention $S_{0}$ is the simple right $B$-module corresponding to $e_{0}^{\prime} B$, so that the $k$-dual $D S_{0}$ is the simple left $B$-module corresponding to $B e_{0}^{\prime}$. It follows that $\operatorname{Hom}_{B \text { op }}\left(B e_{0}^{\prime}, D S_{0}\right) \neq 0$.

We first claim that $B e_{0}^{\prime}$ is a summand of ${ }_{B} T$. By construction, it is clear that $B e_{0}^{\prime}=$ $\operatorname{Hom}_{A}\left(e_{0} A, T\right)$ as left $B$-modules. As in [4, page 33], the functor

$$
{ }^{\star}(-):=\operatorname{Hom}_{A}(-, T): \bmod A \rightarrow \bmod B^{\text {op }}
$$

clearly takes $A_{A} \mapsto{ }_{B} T$, and thus, since $e_{0} A$ is a summand of $A_{A}$, by applying ${ }^{\star}(-)$ we see that ${ }^{\star}\left(e_{0} A\right) \cong B e_{0}^{\prime}$ is a summand of ${ }_{B} T$. 
Now, by [5, VI.5.1] there is an isomorphism

$$
D\left(S_{0} \otimes_{B} B e_{0}^{\prime}\right) \cong \operatorname{Hom}_{B \text { op }}\left(B e_{0}^{\prime}, D S_{0}\right),
$$

which is nonzero by the above. Thus $S_{0} \otimes_{B} B e_{0}^{\prime} \neq 0$. Since, by the above, $S_{0} \otimes_{B} T$ has summand $S_{0} \otimes_{B} B e_{0}^{\prime}$, it follows that $S_{0} \otimes_{B} T \neq 0$. Hence $S_{0} \notin \mathcal{X}_{\alpha}$, so again since $S_{0}$ is simple, necessarily $S_{0} \in \mathcal{Y}_{\alpha}$.

Corollary 5.6 Under Setup 4.1, suppose that $\alpha: C \rightarrow D$ is an atom. If $N \in \mathcal{F}_{\alpha}$ is nonzero, then there exists some $j \neq 0$ such that $\alpha$ starts with $s_{j}$ and, further, $\operatorname{Hom}_{\Lambda_{C}}\left(S_{j}, N\right) \neq 0$.

Proof Certainly $N$ is filtered by simples, so there exists some $0 \leq j \leq n$ with $S_{j} \hookrightarrow N$. In particular, $\operatorname{Hom}\left(S_{j}, N\right) \neq 0$. Since $\mathcal{F}_{\alpha}$ is closed under submodules $S_{j} \in \mathcal{F}_{\alpha}$, and so, by Lemma 5.5, necessarily $j \neq 0$. The result then follows from Lemma 5.4(1).

\section{Proof of faithfulness}

Keeping the notation in the previous sections, under the flops setup of Setup 4.1, recall from Notation 5.2 that every chamber $D$ has an associated algebra $\Lambda_{D}$ and simple modules $S_{0}, S_{1}, \ldots, S_{n}$, and we set $\mathcal{S}:=\bigoplus_{i=0}^{n} S_{i}$. As in the conventions, we write $[a, b]_{t}=\operatorname{Hom}_{\mathrm{D}^{\mathrm{b}}\left(\Lambda_{D}\right)}(a, b[t])$. Although the $D$ is suppressed in this notation, it will be clear from the context in which category to view $\mathcal{S}$.

We will reduce to a key technical lemma in Proposition 6.3, which is an analogue of [3, Proposition 3.1]. The key point of Brav and Thomas is to first find an object $b$ such that

$$
[\mathcal{S}, b]_{\geq d+1}=0
$$

where $d=\operatorname{dim} \Re$. For this there are many choices. To ensure that the method below can be used in future papers to cover situations where $\Lambda$ has infinite global dimension (or flopping contractions $U \rightarrow$ Spec $\Re$ where $U$ need not be smooth), throughout we choose $b=\Lambda$, as is justified in the following lemma.

Lemma 6.1 Suppose that $\mathfrak{R}$ is a $d$-dimensional complete local Gorenstein ring, and that $\Lambda \cong \operatorname{End}_{\mathfrak{R}}(M)$ for some $M \in \operatorname{ref} \mathfrak{R}$, with $\Lambda \in \mathrm{CM} \mathfrak{R}$ (that is, $\Lambda$ is a modifying $\mathfrak{R}$-algebra). Then $b:=\Lambda$ satisfies

$$
\left[S_{i}, b\right]_{d} \neq 0 \quad \text { for all } 0 \leq i \leq n \quad \text { and } \quad[\mathcal{S}, b]_{\geq d+1}=0 .
$$


Proof We know that $\operatorname{Ext}_{\Lambda}^{t}\left(S_{i}, b\right):=\operatorname{Ext}_{\Lambda}^{t}\left(S_{i}, \Lambda\right) \cong \operatorname{Ext}_{\mathfrak{R}}^{t}\left(S_{i}, \mathfrak{R}\right)$, where the last isomorphism is $[15,3.4(5)]$. Hence, by local duality,

$$
\operatorname{depth}_{\mathfrak{R}} S_{i}=d-\sup \left\{t \geq 0 \mid\left[S_{i}, b\right]_{t} \neq 0\right\} .
$$

Clearly, being of finite length, $\operatorname{depth}_{\mathfrak{R}} S_{i}=0$, so we deduce that (6.B) holds.

\subsection{The main result}

Throughout this subsection we will work under the setting of Setup 4.1, and write $b:=\Lambda$. The initial step requires the following elementary lemma:

Lemma 6.2 Suppose that $0 \neq N \in \mathrm{fl} \Lambda_{D}$.

(1) If $y \in \mathrm{D}^{\mathrm{b}}\left(\bmod \Lambda_{D}\right)$ is such that $[\mathcal{S}, y]_{\geq p}=0$, then $[N, y]_{\geq p}=0$.

(2) $[N, b]_{d} \neq 0$ and $[N, b]_{\geq d+1}=0$.

Proof (1) This is an easy induction on the length of the filtration of $N$, using the long exact sequence from $[-, y]$.

(2) By Lemma 6.1, $[\mathcal{S}, b]_{\geq d+1}=0$, so the second statement is a consequence of (1). The first also follows by an induction on the length of the filtration of $N$, using $\left[S_{i}, b\right]_{d} \neq 0$ and $\left[S_{i}, b\right]_{d+1}=0$ for all $0 \leq i \leq n$.

Now, for $\alpha \in \mathbb{G}_{\mathcal{H}}^{+}$, we can decompose $\alpha$ into length-one atoms $\alpha=s_{i_{n}} \cdots s_{i_{1}}$ and define $t_{\alpha}:=t_{i_{n}} \circ \cdots \circ t_{i_{1}}$ (where the $t_{i_{t}}$ are as defined in Notation 4.3), or alternatively we can decompose $\alpha$ into Deligne normal form $\alpha=\alpha_{k} \cdots \alpha_{1}$ and define $t_{\alpha}:=t_{\alpha_{k}} \circ \cdots \circ t_{\alpha_{1}}$ (where the $t_{\alpha_{i}}$ are also as defined in Notation 4.3). The crucial point in the proof of faithfulness is that by Theorem 4.6(2) these yield the same functor.

The following is our analogue of the main technical lemma of Brav and Thomas [3, Proposition 3.1]. Using torsion pairs, the proof only needs to induct on the number of Deligne factors, whereas Brav and Thomas use a more complicated double induction.

Proposition 6.3 Let $1 \neq \alpha \in \mathbb{G}_{\mathcal{H}}^{+}$have Deligne normal form $\alpha=\alpha_{k} \circ \cdots \circ \alpha_{1}$. Then:

(1) $\left[\mathcal{S}, t_{\alpha} b\right]_{\geq k+d+1}=0$.

(2) $\left[S_{i}, t_{\alpha} b\right]_{k+d} \neq 0$ if and only if $i \neq 0$ and the atom $\alpha_{k}$ ends (up to the relations in $\mathbb{G}_{\mathcal{H}}^{+}$) by passing through wall $i$. In particular, $\left[\mathcal{S}, t_{\alpha} b\right]_{k+d} \neq 0$.

(3) The maximal $p$ such that $\left[\mathcal{S}, t_{\alpha} b\right]_{p} \neq 0$ is precisely $p=k+d$. 
Proof Statement (3) follows immediately from (1) and (2), so we prove both (1) and (2) together using induction on the number of Deligne factors.

Base case $\left(k=1\right.$, ie $\alpha$ is an atom) Since $S_{i}$ is simple, there are only two cases, namely $S_{i} \in \mathcal{Y}_{\alpha}$ or $S_{i} \in \mathcal{X}_{\alpha}$, and using Lemmas 5.4 and 5.5 we can characterise these:

(a) ( $S_{i} \in \mathcal{Y}_{\alpha}$; equivalently, $i=0$, or $i \neq 0$ and $\alpha$ does not end with $s_{i}$ ) By (5.B), $t_{\alpha}^{-1}\left(S_{i}\right) \cong N$ for some finite-length module $N$. Hence, by Lemma 6.2(2),

$$
\left[S_{i}, t_{\alpha} b\right]_{\geq d+1}=[N, b]_{\geq d+1}=0 .
$$

(b) $\left(S_{i} \in \mathcal{X}_{\alpha}\right.$; equivalently, $i \neq 0$ and $\alpha$ ends with $\left.s_{i}\right)$ By (5.B), $t_{\alpha}^{-1}\left(S_{i}\right) \cong N[1]$ for some finite-length module $N$. Hence, again by Lemma 6.2(2),

$$
\left[S_{i}, t_{\alpha} b\right]_{\geq d+2}=[N[1], b]_{\geq d+2}=[N, b]_{\geq d+1}=0
$$

and

$$
\left[S_{i}, t_{\alpha} b\right]_{d+1}=[N, b]_{d} \neq 0 .
$$

Combining (a) and (b) proves (1)-(2) in the case $k=1$.

Induction step We assume that the result is true for all paths with at most $k-1$ Deligne factors. Write $\alpha=\alpha_{k} \circ \beta$, where $\beta:=\alpha_{k-1} \circ \cdots \circ \alpha_{1}$. By induction,

$$
\left[\mathcal{S}, t_{\beta} b\right]_{\geq k+d}=0
$$

and $\left[S_{j}, t_{\beta} b\right]_{k+d-1} \neq 0$ if and only if $j \neq 0$ and $\alpha_{k-1}$ ends with $s_{j}$. Again there are only two cases:

(a) $\left(S_{i} \in \mathcal{Y}_{\alpha_{k}}\right.$; equivalently, $i=0$, or $i \neq 0$ and $\alpha_{k}$ does not end with $s_{i}$ ) By (5.B), $t_{\alpha_{k}}^{-1}\left(S_{i}\right) \cong N$ for some finite-length module $N$. Hence,

$$
\left[S_{i}, t_{\alpha} b\right]_{\geq k+d}=\left[t_{\alpha_{k}}^{-1} S_{i}, t_{\beta} b\right]_{\geq k+d}=\left[N, t_{\beta} b\right]_{\geq k+d}=0 \text { by Lemma 6.2(1). }
$$

(b) $\left(S_{i} \in \mathcal{X}_{\alpha_{k}}\right.$; equivalently, $i \neq 0$ and $\alpha_{k}$ ends with $s_{i}$ ) By (5.B), $t_{\alpha_{k}}^{-1}\left(S_{i}\right) \cong N[1]$ for some finite-length module $N$. Thus,

$$
\left[S_{i}, t_{\alpha} b\right]_{\geq k+d+1}=\left[N[1], t_{\beta} b\right]_{\geq k+d+1}=\left[N, t_{\beta} b\right]_{\geq k+d}=0 \quad \text { by Lemma 6.2(1). }
$$

Similarly,

$$
\left[S_{i}, t_{\alpha} b\right]_{k+d}=\left[N[1], t_{\beta} b\right]_{k+d}=\left[N, t_{\beta} b\right]_{k+d-1},
$$

so it remains to show that $\left[N, t_{\beta} b\right]_{k+d-1} \neq 0$. But, by Corollary 5.6, there exists $j \neq 0$ such that $\alpha_{k}$ starts with $s_{j}$, and $S_{j} \hookrightarrow N$. Write $C$ for the cokernel, which necessarily 
has finite length, and consider the long exact sequence

$$
\cdots \rightarrow\left[C, t_{\beta} b\right]_{k+d-1} \rightarrow\left[N, t_{\beta} b\right]_{k+d-1} \rightarrow\left[S_{j}, t_{\beta} b\right]_{k+d-1} \rightarrow\left[C, t_{\beta} b\right]_{k+d}=0 .
$$

Since $\alpha_{k}$ starts with $s_{j}$, necessarily $\alpha_{k-1}$ ends with $s_{j}$, otherwise $s_{j} \circ \alpha_{k-1}$ is an atom by Lemma 5.1, which would contradict the fact that $\alpha_{k} \circ \alpha_{k-1} \circ \cdots \circ \alpha_{1}$ is in Deligne normal form. Thus, $\left[S_{j}, t_{\beta} b\right]_{k+d-1} \neq 0$ by the inductive hypothesis. It follows that $\left[N, t_{\beta} b\right]_{k+d-1} \neq 0$.

Combining (a) and (b) proves (1)-(2) in the case of $k$ factors, so by induction the result follows.

The remainder of the proof of faithfulness is straightforward.

Definition 6.4 Define the groupoid $\mathcal{G}_{\Lambda}$ as follows:

(1) The vertices are $\mathrm{D}^{\mathrm{b}}\left(\bmod \Lambda_{C}\right)$ for chambers $C$ of $\mathcal{H}$.

(2) The morphisms between any two vertices are all triangle equivalences between the corresponding derived categories.

By Theorem 4.6(2) and Remark 4.7, there is a natural functor

$$
F_{\Lambda}: \mathbb{G}_{\mathcal{H}} \rightarrow \mathcal{G}_{\Lambda}
$$

which sends a simple wall crossing $s_{i}$ to the corresponding equivalence $t_{i}$.

Theorem 6.5 The functor $F_{\Lambda}$ is faithful.

Proof This is an easy induction. We use Lemma 2.11, so suppose that

$$
t_{\alpha}=t_{\beta}: \mathrm{D}^{\mathrm{b}}\left(\bmod \Lambda_{C}\right) \rightarrow \mathrm{D}^{\mathrm{b}}\left(\bmod \Lambda_{D}\right)
$$

for some $\alpha, \beta \in \mathbb{G}_{\mathcal{H}}^{+}$. Since $t_{\alpha}=t_{\beta}$, we deduce from Proposition 6.3(3) that $\alpha$ and $\beta$ have the same number of Deligne factors, so write

$$
\alpha=\alpha_{k} \cdots \alpha_{1} \text { and } \beta=\beta_{k} \cdots \beta_{1}
$$

in Deligne normal form. By induction, it is enough to show that $\alpha_{k}=\beta_{k}$ and $t_{\alpha_{k-1} \cdots \alpha_{1}}=$ $t_{\beta_{k-1} \cdots \beta_{1}}$. We may assume that $\ell:=\ell\left(\alpha_{k}\right) \leq \ell\left(\beta_{k}\right)$. By Proposition 6.3(2), since $t_{\alpha}=t_{\beta}$, both $\alpha_{k}$ and $\beta_{k}$ end with the same simple wall crossing, say $s_{i_{1}}$, so we can write $\alpha_{k}=s_{i_{1}} \widetilde{\alpha}_{k}$ and $\beta_{k}=s_{i_{1}} \widetilde{\beta}_{k}$. Hence, applying $t_{i_{1}}^{-1}$ to $t_{\alpha}=t_{\beta}$, we deduce that $t_{\widetilde{\alpha}_{k} \alpha_{k-1} \cdots \alpha_{1}}=t_{\widetilde{\beta}_{k} \beta_{k-1} \cdots \beta_{1}}$. 
Repeating the above argument, we can write $\alpha_{k}=s_{i_{1}} \cdots s_{i_{\ell}}$ and $\beta_{k}=s_{i_{1}} \cdots s_{i_{\ell}} \gamma$ for some $\gamma \in \mathbb{G}_{\mathcal{H}}^{+}$, and so we have $t_{\alpha_{k-1} \cdots \alpha_{1}}=t_{\gamma \beta_{k-1} \cdots \beta_{1}}$. But, again by Proposition 6.3(3), $\gamma$ must be a length-zero path. Hence, we have $\alpha_{k}=s_{i_{1}} \cdots s_{i_{\ell}}=\beta_{k}$ and $t_{\alpha_{k-1} \cdots \alpha_{1}}=$ $t_{\beta_{k-1} \cdots \beta_{1}}$, as required.

Corollary 6.6 For every chamber $C$, the induced map

$$
\pi_{1}\left(\mathbb{C}^{n} \backslash \mathcal{H}_{\mathbb{C}}\right) \rightarrow \text { Auteq } \mathrm{D}^{\mathrm{b}}\left(\bmod \Lambda_{C}\right)
$$

is an injective group homomorphism

Proof By Corollary 2.12, this follows immediately from Theorem 6.5.

\subsection{Geometric corollaries}

Although the above results were stated in the formal fibre setting, they easily imply the following global results:

Corollary 6.7 Suppose that $f: X \rightarrow X_{\text {con }}$ is a flopping contraction between 3-folds, where $X$ is smooth and all curves in the contraction $f$ are individually floppable. Then there is an injective group homomorphism

$$
\varphi: \pi_{1}\left(\mathbb{C}^{n} \backslash \mathcal{H}_{\mathbb{C}}\right) \rightarrow \text { Auteq } \mathrm{D}^{\mathrm{b}}(\operatorname{coh} X) .
$$

Proof As in [10, Proposition 6.2], the functors in the image of $\varphi$ fix the skyscraper sheaves away from the flopping curves. Hence, the relations can be detected on the formal fibre, where the result is Corollary 6.6.

In the case when the $n$ curves are not individually floppable, there is still a group action, but only by a subgroup $S$ of $\pi_{1}\left(\mathbb{C}^{n} \backslash \mathcal{H}_{\mathbb{C}}\right)$ defined to be the subgroup generated by the $J$-twists of [10], where $J$ runs through all subsets of $\{1, \ldots, n\}$. The proof of faithfulness extends to this case too.

Corollary 6.8 Suppose that $f: X \rightarrow X_{\text {con }}$ is a flopping contraction between 3-folds, where $X$ is smooth. Then there is an injective group homomorphism

$$
S \rightarrow \text { Auteq } \mathrm{D}^{\mathrm{b}}(\operatorname{coh} X) .
$$

Proof Again, by [10, Proposition 6.2], the functors in the image of the above homomorphism fix the skyscraper sheaves away from the flopping curves. Hence, the 
relations can be detected on the formal fibre. Since there $\pi_{1}\left(\mathbb{C}^{n} \backslash \mathcal{H}_{\mathbb{C}}\right)$ acts faithfully by Corollary 6.6, so does any subgroup.

Recall that if $\mathcal{A}$ is the heart of a bounded $t$-structure on a triangulated category $\mathcal{D}$, and $\mathcal{A}$ admits a torsion pair $(\mathcal{T}, \mathcal{F})$, then the tilt of $\mathcal{A}$ with respect to this torsion pair is defined to be

$$
\mathcal{A}^{\sharp}:=\left\{E \in \mathcal{D} \mid H^{i}(E)=0 \text { for } i \notin\{-1,0\}, H^{-1}(E) \in \mathcal{F} \text { and } H^{0}(E) \in \mathcal{T}\right\} .
$$

By [12, Proposition I.2.1], $\mathcal{A}^{\#}$ is also the heart of a bounded $t$-structure on $\mathcal{D}$.

Now, for a 3-fold flopping contraction $f: X \rightarrow X_{\text {con }}$, consider the full subcategories

$$
\begin{aligned}
& \mathcal{T}_{0}:=\left\{T \in \operatorname{coh} X \mid \boldsymbol{R}^{1} f_{*}(T)=0\right\}, \\
& \mathcal{F}_{0}:=\left\{F \in \operatorname{coh} X \mid f_{*}(F)=0, \operatorname{Hom}(\mathcal{C}, F)=0\right\},
\end{aligned}
$$

where $\mathcal{C} \subset \operatorname{coh} X$ is the full subcategory consisting of objects $E$ such that $\boldsymbol{R} f_{*}(E)=0$. Then $\left(\mathcal{T}_{0}, \mathcal{F}_{0}\right)$ is a torsion pair by [1, Lemma 3.1.2], and the category of perverse sheaves relative to $f$ is defined to be

$$
{ }^{0} \operatorname{Per}\left(X, X_{\text {con }}\right):=(\operatorname{coh} X)^{\#},
$$

namely the tilt of the standard heart $\operatorname{coh} X \subset \mathrm{D}^{\mathrm{b}}(\operatorname{coh} X)$ with respect to the torsion pair $\left(\mathcal{T}_{0}, \mathcal{F}_{0}\right)$.

The following is a further consequence of the results in this paper, and may be of independent interest. The first part is implicit in [10]; the second part is new.

Theorem 6.9 Consider two crepant resolutions

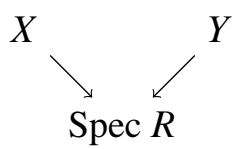

of Spec $R$, where $R$ is an isolated $c D V$ singularity.

(1) Given two minimal chains of flops connecting $X$ and $Y$, the composition of flop functors associated to each chain are functorially isomorphic.

(2) Perverse sheaves on $Y$, namely ${ }^{0} \operatorname{Per}(Y, R)$, can be obtained from perverse sheaves on $X$, namely ${ }^{0} \operatorname{Per}(X, R)$, by a single tilt at a torsion pair. 
Proof (1) By [7, 1.10, 1.12], any two minimal paths can be identified provided that in the Deligne groupoid the codimension-two relations hold. By [10, 3.20], the codimension-two relations precisely correspond to the braiding of the 2-curve flop functors, which is proved in [10, 3.9, 3.20].

(2) Consider a minimal path of flops

$$
\mathrm{D}^{\mathrm{b}}(\operatorname{coh} X) \stackrel{\mathrm{F}_{i_{1}}}{\longrightarrow} \mathrm{D}^{\mathrm{b}}\left(\operatorname{coh} X_{i_{1}}\right) \stackrel{\mathrm{F}_{i_{2}}}{\longrightarrow} \cdots \stackrel{\mathrm{F}_{i_{n}}}{\longrightarrow} \mathrm{D}^{\mathrm{b}}(\operatorname{coh} Y)
$$

connecting $X$ and $Y$. By [1], $X$ is derived equivalent to $\operatorname{End}_{R}(M)$, say, and $Y$ is derived equivalent to $\operatorname{End}_{R}(N)$, say, and under this identification ${ }^{0} \operatorname{Per}(X, R)$ corresponds to $\bmod \operatorname{End}_{R}(M)$, and ${ }^{0} \operatorname{Per}(Y, R)$ corresponds to $\bmod \operatorname{End}_{R}(N)$. Hence, it suffices to show that $\bmod _{R} \operatorname{End}_{R}(N)$ can be obtained from $\bmod _{R}(M)$ by a tilt at a torsion pair.

Consider $T:=\operatorname{Hom}_{R}(M, N)$. This is a tilting $\operatorname{End}_{R}(M)-$ module, by [17, 4.17]. But, since $\operatorname{End}_{R}(M)$ is noetherian,

$$
\begin{aligned}
& \mathcal{T}:=\left\{X \in \bmod \operatorname{End}_{R}(M) \mid \operatorname{Ext}_{\operatorname{End}_{R}(M)}^{1}(T, X)=0\right\}, \\
& \mathcal{F}:=\left\{X \in \bmod \operatorname{End}_{R}(M) \mid \operatorname{Hom}_{\operatorname{End}_{R}(M)}(T, X)=0\right\}
\end{aligned}
$$

gives a torsion pair $(\mathcal{T}, \mathcal{F})$ on $\bmod _{\operatorname{End}}(M)$; the proof is identical to $[27,2.7(3)]$. Using the finitely generated version of the equivalences (5.A), it is then clear that $\bmod \operatorname{End}_{R}(N)$ is obtained from $\bmod \operatorname{End}_{R}(M)$ by tilting at $(\mathcal{T}, \mathcal{F})$.

\section{Appendix A: Brav and Thomas revisited}

In this appendix, which can be read independently of the previous sections, we give a direct proof of the faithfulness of the braid action on the minimal resolution of Kleinian singularities, just to demonstrate that our torsion pairs viewpoint simplifies the [3] proof. Thus in this section we consider the minimal resolution $X \rightarrow \operatorname{Spec} R$ of a Kleinian singularity, let $\Lambda$ denote the completion of the preprojective algebra of the corresponding extended Dynkin diagram, and set $b:=\mathcal{S}$, where $\mathcal{S}$ is the direct sum of the vertex simples $S_{0}, S_{1}, \ldots, S_{n}$.

The initial step requires the following elementary lemma, which replaces Lemma 6.2.

Lemma A.1 Suppose that $M \in \mathrm{fl} \Lambda$.

(1) If $y \in \mathrm{D}^{\mathrm{b}}(\bmod \Lambda)$ is such that $[\mathcal{S}, y]_{\geq p}=0$, then $[M, y]_{\geq p}=0$.

(2) $[M, \mathcal{S}]_{2} \neq 0$ and $[M, \mathcal{S}]_{\geq 3}=0$. 
Proof (1) This is an easy induction on the length of the filtration of $M$, using the long exact sequence from $[-, y]$.

(2) Since $\Lambda$ is $2-\mathrm{CY},[\mathcal{S}, \mathcal{S}]_{\geq 3}=0$, so the second statement is a consequence of (1). The first also follows by an induction on the length of the filtration of $M$, using the fact that $\left[S_{i}, \mathcal{S}\right]_{2} \neq 0$ for all $0 \leq i \leq n$.

For every primitive idempotent $e_{i}$ corresponding to a vertex of the extended Dynkin diagram, following [15, Section 6] we set

$$
I_{i}:=\Lambda\left(1-e_{i}\right) \Lambda
$$

It is known by [11, Section 6] that $\operatorname{RHom}_{\Lambda}\left(I_{i},-\right)$ is functorially isomorphic to the twist functor $t_{i}$. To control iterations, for any $\alpha \in W$, where $W$ is the associated Weyl group, choose a reduced expression $\alpha=s_{i_{n}} \circ \cdots \circ s_{i_{1}}$ and define

$$
I_{\alpha}:=I_{i_{n}} \cdots I_{i_{1}}
$$

Since the expression is reduced,

$$
I_{\alpha} \cong I_{i_{n}} \otimes_{\Lambda}^{L} \cdots \otimes_{\Lambda}^{L} I_{i_{1}}
$$

by $[27,2.21]$, so that

$$
\text { (A.A) } \quad t_{\alpha}:=\operatorname{RHom}_{\Lambda}\left(I_{\alpha},-\right) \cong t_{i_{n}} \circ \cdots \circ t_{i_{1}}
$$

By the usual torsion pair associated to a tilting module, as in [27, 2.9] and Section 5, for any vertex simple $S_{i}$, either $S_{i} \in \mathcal{X}_{\alpha}$ or $S_{i} \in \mathcal{Y}_{\alpha}$, where

$$
\begin{aligned}
& \mathcal{X}_{\alpha}:=\left\{N \in \mathrm{fl} \Lambda \mid N \otimes_{\Lambda} I_{\alpha}=0\right\}, \\
& \mathcal{Y}_{\alpha}:=\left\{N \in \mathrm{fl} \Lambda \mid \operatorname{Tor}_{1}^{\Lambda}\left(N, I_{\alpha}\right)=0\right\},
\end{aligned}
$$

and furthermore the equivalence (A.A) forces

$$
t_{\alpha}^{-1}\left(S_{i}\right)= \begin{cases}\operatorname{Tor}_{1}\left(S_{i}, I_{\alpha}\right)[1] & \text { if } S_{i} \in \mathcal{X}_{\alpha}, \\ S_{i} \otimes I_{\alpha} & \text { if } S_{i} \in \mathcal{Y}_{\alpha} .\end{cases}
$$

There is a corresponding version of Lemmas 5.4 and 5.5 and Corollary 5.6, which we will use freely below, since these were already very well known $[27,2.28,5.4]$ in the preprojective algebra setting. With this, we can now prove the main technical lemma [3, Proposition 3.1] in the setting of minimal resolutions of Kleinian singularities.

Proposition A.2 Let $1 \neq \alpha \in \mathbb{G}_{\mathcal{H}}^{+}$have Deligne normal form $\alpha=\alpha_{k} \circ \cdots \circ \alpha_{1}$. Then: (1) $\left[\mathcal{S}, t_{\alpha} \mathcal{S}\right]_{\geq k+3}=0$. 
(2) $\left[S_{i}, t_{\alpha} \mathcal{S}\right]_{k+2} \neq 0$ if and only if $i \neq 0$ and the atom $\alpha_{k}$ ends (up to the relations in $\mathbb{G}_{\mathcal{H}}^{+}$) by passing through wall $i$. In particular, $\left[\mathcal{S}, t_{\alpha} \mathcal{S}\right]_{k+2} \neq 0$.

(3) The maximal $p$ such that $\left[\mathcal{S}, t_{\alpha} \mathcal{S}\right]_{p} \neq 0$ is precisely $p=k+2$.

Proof Statement (3) follows immediately from (1) and (2), so we prove both (1) and (2) together using induction on the number of Deligne factors.

Base case $\left(k=1\right.$, ie $\alpha$ is an atom) Since $S_{i}$ is simple, there are only two cases, namely $S_{i} \in \mathcal{Y}_{\alpha}$ or $S_{i} \in \mathcal{X}_{\alpha}$, and using Lemmas 5.4 and 5.5 we can characterise these:

(a) $\left(S_{i} \in \mathcal{Y}_{\alpha}\right.$; equivalently, $i=0$, or $i \neq 0$ and $\alpha$ does not end with $s_{i}$ ) By (A.B), $t_{\alpha}^{-1}\left(S_{i}\right) \cong M$ for some finite-length module $M$. Hence, by Lemma A.1(2),

$$
\left[S_{i}, t_{\alpha} \mathcal{S}\right]_{\geq 3}=[M, \mathcal{S}]_{\geq 3}=0 .
$$

(b) $\left(S_{i} \in \mathcal{X}_{\alpha}\right.$; equivalently, $i \neq 0$ and $\alpha$ ends with $\left.s_{i}\right)$ By (A.B), $t_{\alpha}^{-1}\left(S_{i}\right) \cong M[1]$ for some finite-length module $M$. Hence, again by Lemma A.1(2),

$$
\left[S_{i}, t_{\alpha} \mathcal{S}\right]_{\geq 4}=[M[1], \mathcal{S}]_{\geq 4}=[M, \mathcal{S}]_{\geq 3}=0
$$

and

$$
\left[S_{i}, t_{\alpha} \mathcal{S}\right]_{3}=[M, \mathcal{S}]_{2} \neq 0 \text {. }
$$

Combining (a) and (b) proves (1)-(2) in the case $k=1$.

Induction step We assume that the result is true for all paths with at most $k-1$ Deligne factors. Write $\alpha=\alpha_{k} \circ \beta$, where $\beta:=\alpha_{k-1} \circ \cdots \circ \alpha_{1}$. By induction,

$$
\left[\mathcal{S}, t_{\beta} \mathcal{S}\right]_{\geq k+2}=0
$$

and $\left[S_{j}, t_{\beta} \mathcal{S}\right]_{k+1} \neq 0$ if and only if $j \neq 0$ and $\alpha_{k-1}$ ends with $s_{j}$. Again there are only two cases:

(a) $\left(S_{i} \in \mathcal{Y}_{\alpha_{k}}\right.$; equivalently, $i=0$, or $i \neq 0$ and $\alpha_{k}$ does not end with $s_{i}$ ) By (A.B), $t_{\alpha_{k}}^{-1}\left(S_{i}\right) \cong M$ for some finite-length module $M$. Hence,

$$
\left[S_{i}, t_{\alpha} \mathcal{S}\right]_{\geq k+2}=\left[t_{\alpha_{k}}^{-1} S_{i}, t_{\beta} \mathcal{S}\right]_{\geq k+2}=\left[M, t_{\beta} \mathcal{S}\right]_{\geq k+2}=0 \quad \text { by Lemma A.1(1). }
$$

(b) $\left(S_{i} \in \mathcal{X}_{\alpha_{k}}\right.$; equivalently, $i \neq 0$ and $\alpha_{k}$ ends with $s_{i}$ ) By (A.B), $t_{\alpha_{k}}^{-1}\left(S_{i}\right) \cong M[1]$ for some finite-length module $M$. Thus,

$$
\left[S_{i}, t_{\alpha} \mathcal{S}\right]_{\geq k+3}=\left[M[1], t_{\beta} \mathcal{S}\right]_{\geq k+3}=\left[M, t_{\beta} \mathcal{S}\right]_{\geq k+2}=0 \quad \text { by Lemma A.1(1). }
$$

Similarly,

$$
\left[S_{i}, t_{\alpha} \mathcal{S}\right]_{k+2}=\left[M[1], t_{\beta} \mathcal{S}\right]_{k+2}=\left[M, t_{\beta} \mathcal{S}\right]_{k+1}
$$


so it remains to show that $\left[M, t_{\beta} \mathcal{S}\right]_{k+1} \neq 0$. But, by Corollary 5.6, there exists $j \neq 0$ such that $\alpha_{k}$ starts at $s_{j}$, and $S_{j} \hookrightarrow M$. Write $C$ for the cokernel, which necessarily has finite length, and consider the long exact sequence

$$
\cdots \rightarrow\left[C, t_{\beta} \mathcal{S}\right]_{k+1} \rightarrow\left[M, t_{\beta} \mathcal{S}\right]_{k+1} \rightarrow\left[S_{j}, t_{\beta} \mathcal{S}\right]_{k+1} \rightarrow\left[C, t_{\beta} \mathcal{S}\right]_{k+2}=0
$$

Since $\alpha_{k}$ starts with $s_{j}$, necessarily $\alpha_{k-1}$ ends with $s_{j}$, otherwise $s_{j} \circ \alpha_{k-1}$ is an atom by Lemma 5.1, which would contradict the fact that $\alpha_{k} \circ \alpha_{k-1} \circ \cdots \circ \alpha_{1}$ is in Deligne normal form. Thus, $\left[S_{j}, t_{\beta} \mathcal{S}\right]_{k+1} \neq 0$ by the inductive hypothesis. It follows that $\left[M, t_{\beta} \mathcal{S}\right]_{k+1} \neq 0$.

Combining (a) and (b) proves (1)-(2) in the case of $k$ factors, so by induction the result follows.

From here, the proof of faithfulness follows exactly as in [3, Theorem 3.1]. Alternatively, we can use Lemma 2.11 as in Theorem 6.5 to deduce that the groupoid action is faithful. Since $B_{\Gamma} \cong \pi_{1}\left(\left(\mathbb{C}^{n} \backslash \mathcal{H}_{\mathbb{C}}\right) / W_{\Gamma}\right)$, and each vertex of $\mathcal{G}$ is by definition the same $\mathrm{D}^{\mathrm{b}}(\operatorname{coh} X)$, as is standard by identifying all vertices we can simply reinterpret the faithful groupoid action as an injective group homomorphism $B_{\Gamma} \rightarrow \operatorname{Auteq}^{\mathrm{b}}(\operatorname{coh} X)$.

\section{Appendix B: Tilting background}

In this appendix, which is logically independent of all other sections, we give some known tilting results that were used in the text, and we also prove Lemma 3.4 and Theorem 3.5.

Throughout $\Lambda$ is a basic $\mathfrak{R}$-algebra, where $\mathfrak{R}$ is a complete local domain. Recall that if $T \in$ tilt $_{0} \Lambda$ and its mutation $\nu_{i} T$ at a direct summand $T_{i}$ exists, either there is an exact sequence

$$
0 \rightarrow T_{i} \stackrel{f}{\longrightarrow} T^{\prime} \rightarrow U_{i} \rightarrow 0
$$

where $f$ is a minimal left $\operatorname{add}\left(T / T_{i}\right)$-approximation, or an exact sequence

$$
0 \rightarrow U_{i} \rightarrow T^{\prime} \stackrel{g}{\longrightarrow} T_{i} \rightarrow 0,
$$

where $g$ is a minimal right $\operatorname{add}\left(T / T_{i}\right)$-approximation. By definition, $T>v_{i} T$ in the former case, and $T<v_{i} T$ in the latter case.

Suppose that $T \in$ tilt $\Lambda$ with $\operatorname{End}_{\Lambda}(T) \cong \Gamma$. By projectivisation, the indecomposable summands of $\Gamma$ correspond to the indecomposable summands of $T$. Hence, we can 
try to mutate $T \in$ tilt $\Lambda$ to form $\nu_{i} T$, and similarly we can try to mutate $\Gamma \in$ tilt $\Gamma$ to form $v_{i} \Gamma$. Although the following is elementary and is known to experts, references to the literature only exist when $\bmod \Lambda$ is Hom-finite, so here we give the proof in full.

Proposition B.1 Suppose that $T \in$ tilt $\Lambda$, and set $\Gamma:=\operatorname{End}_{\Lambda}(T)$. If $v_{i} T$ exists and, further, $T>v_{i} T$, then $v_{i} \Gamma \in$ tilt $\Gamma$ exists, there is an isomorphism $v_{i} T \cong v_{i} \Gamma \otimes_{\Gamma}^{\boldsymbol{L}} T$ in $\mathrm{D}^{\mathrm{b}}(\bmod \Lambda)$, and, further, $v_{i} T \in \mathcal{T}:=\left\{N \in \bmod \Lambda \mid \operatorname{Ext}_{\Lambda}^{1}(T, N)=0\right\}$.

Proof To ease notation write $V:=T / T_{i}$.

Since $v_{i} T=V \oplus U_{i}$ exists and $T>v_{i} T$, as above there exists an exact sequence

$$
0 \rightarrow T_{i} \stackrel{f}{\longrightarrow} T^{\prime} \rightarrow U_{i} \rightarrow 0,
$$

where $f$ is a minimal left add $V$-approximation. Applying $\operatorname{Hom}_{\Lambda}(T,-)$ gives an exact sequence

$$
0 \rightarrow \operatorname{Hom}_{\Lambda}\left(T, T_{i}\right) \stackrel{f \circ}{\longrightarrow} \operatorname{Hom}_{\Lambda}\left(T, T^{\prime}\right) \rightarrow \operatorname{Hom}_{\Lambda}\left(T, U_{i}\right) \rightarrow 0 .
$$

Write $\Gamma=\operatorname{Hom}_{\Lambda}(T, T)=\operatorname{Hom}_{\Lambda}(T, V) \oplus \operatorname{Hom}_{\Lambda}\left(T, T_{i}\right):=\Gamma_{V} \oplus \Gamma_{i}$; then, by projectivisation, (B.A) is a projective resolution of $\operatorname{Hom}_{\Lambda}\left(T, U_{i}\right)$. We claim that $(f \circ)$ is a minimal left add $\Gamma_{V}$-approximation. To see this, simply apply $\operatorname{Hom}_{\Gamma}\left(-, \Gamma_{V}\right)$ to (B.A) to obtain a commutative diagram

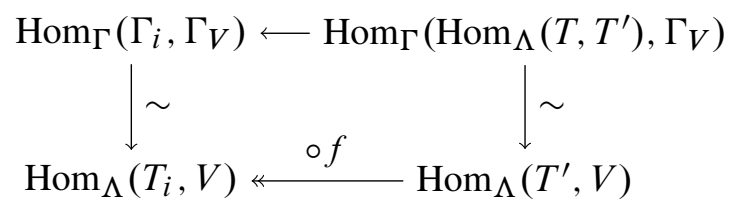

where the vertical maps are isomorphisms by projectivisation, and the bottom map is surjective since $f$ is an add $V$-approximation. It follows that the top map is surjective, and hence $(f \circ)$ is a left add $\Gamma_{V}$-approximation. The minimality of ( $f \circ$ ) follows since the left add $V$-approximation $f$ is minimal, and the functor $\operatorname{Hom}_{\Lambda}(T,-)$ : add $T \rightarrow \operatorname{proj} \Gamma$ is fully faithful.

As is standard $[17,6.6]$, since $(f \circ)$ in (B.A) is injective and an approximation, it follows that $\Gamma_{V} \oplus \operatorname{Hom}_{\Lambda}\left(T, U_{i}\right) \in$ tilt $\Gamma$, and evidently $v_{i} \Gamma \cong \Gamma_{V} \oplus \operatorname{Hom}_{\Lambda}\left(T, U_{i}\right)$ since $\nu_{i} \Gamma$ and $\Gamma$ differ at only one indecomposable summand.

Now, using (B.A) to compute the derived tensor in $\mathrm{D}^{\mathrm{b}}(\bmod \Lambda)$, observe first that 
$\operatorname{Hom}_{\Lambda}\left(T, U_{i}\right) \otimes_{\Gamma}^{\boldsymbol{L}} T \cong \cdots \rightarrow 0 \rightarrow \operatorname{Hom}_{\Lambda}\left(T, T_{i}\right) \otimes_{\Gamma} T \rightarrow \operatorname{Hom}_{\Lambda}\left(T, T^{\prime}\right) \otimes_{\Gamma} T \rightarrow 0 \rightarrow \cdots$

$$
\cong \cdots \rightarrow 0 \rightarrow T_{i} \stackrel{f}{\longrightarrow} T^{\prime} \rightarrow 0 \rightarrow \cdots,
$$

which, since $f$ is injective, is clearly isomorphic to $U_{i}$ (in degree zero). Hence,

$$
\nu_{i} \Gamma \otimes_{\Gamma}^{\boldsymbol{L}} T \cong\left(\operatorname{Hom}_{\Lambda}(T, V) \otimes_{\Gamma} T\right) \oplus\left(\operatorname{Hom}_{\Lambda}\left(T, U_{i}\right) \otimes_{\Gamma}^{\boldsymbol{L}} T\right) \cong V \oplus U_{i},
$$

where $\operatorname{Hom}_{\Lambda}(T, V) \otimes_{\Gamma} T \cong V$ holds since $T$ is tilting and $V$ is projective. It follows that $v_{i} \Gamma \otimes_{\Gamma}^{\boldsymbol{L}} T \cong v_{i} T$ in $\mathrm{D}^{\mathrm{b}}(\bmod \Lambda)$. Applying $\mathbf{R H o m}_{\Lambda}(T,-)$ gives the final statement.

Lemma B.2 (Lemma 3.4) Suppose that $\Lambda$ is a basic $\mathfrak{R}$-algebra, where $\mathfrak{R}$ is a complete local domain. If $T, U \in$ tilt $_{0} \Lambda$ are related by a mutation at an indecomposable summand, then $C_{T}$ and $C_{U}$ do not overlap, and are separated by a codimension-one wall.

Proof By assumption, there are indecomposable modules $T_{0}, \ldots, T_{n}$ and $U_{n}$ such that $T=T_{<n} \oplus T_{n}$ and $U=T_{<n} \oplus U_{n}$, where $T_{<n}:=\bigoplus_{i=0}^{n-1} T_{i}$. We may assume that $T>U$, and then there is an exact sequence

$$
0 \rightarrow T_{n} \rightarrow X_{<n} \rightarrow U_{n} \rightarrow 0,
$$

where $X_{<n} \in$ add $T_{<n}$, say $X_{<n}:=T_{0}^{\oplus a_{0}} \oplus T_{1}^{\oplus a_{1}} \oplus \cdots \oplus T_{n-1}^{\oplus a_{n-1}}$. Thus, recalling that the $[-]$ notation works modulo $\operatorname{Span}\left\{\boldsymbol{e}_{0}\right\}$, we see that

$$
\left[T_{n}\right]=-\left[U_{n}\right]+\sum_{i=1}^{n-1} a_{i}\left[T_{i}\right]
$$

in $\Theta_{\Lambda}$, and so

$$
\begin{aligned}
C_{T} & :=\left\{\sum_{i=1}^{n} \vartheta_{i}\left[T_{i}\right] \mid \vartheta_{i}>0 \text { for all } 1 \leq i \leq n\right\} \\
& =\left\{\sum_{i=1}^{n-1}\left(\vartheta_{i}+a_{i} \vartheta_{n}\right)\left[T_{i}\right]-\vartheta_{n}\left[U_{n}\right] \mid \vartheta_{i}>0 \text { for all } 1 \leq i \leq n\right\} .
\end{aligned}
$$

Since $U$ is tilting, the classes $\left[T_{0}\right], \ldots,\left[T_{n-1}\right]$ and $\left[U_{n}\right]$ of indecomposable summands of $U$ span $K_{0} \otimes_{\mathbb{Z}} \mathbb{R} \cong \mathbb{R}^{n+1}$. Hence, they form a basis of $K_{0} \otimes_{\mathbb{Z}} \mathbb{R}$, and in particular the classes $\left[T_{1}\right], \ldots,\left[T_{n-1}\right]$ and $\left[U_{n}\right]$ in $\Theta_{\Lambda}$ form a basis of $\Theta_{\Lambda}$.

Write $H \subset \Theta_{\Lambda}$ for the linear subspace spanned by $\left[T_{1}\right], \ldots,\left[T_{n-1}\right]$. Then $H$ separates $\Theta_{\Lambda}$ into two halfspaces $H_{+}:=\left\{\sum_{i=1}^{n-1} b_{i}\left[T_{i}\right]+a\left[U_{n}\right] \mid a>0\right\}$ and $H_{-}:=$ $\left\{\sum_{i=1}^{n-1} b_{i}\left[T_{i}\right]+a\left[U_{n}\right] \mid a<0\right\}$. Since $C_{T} \subset H_{-}, C_{U} \subset H_{+}$and $H_{+} \cap H_{-}=\varnothing$, we 
obtain $C_{T} \cap C_{U}=\varnothing$. It is clear that $C_{T}$ and $C_{U}$ are separated by a codimension-one wall contained in $H$.

Lemma B.3 Suppose that $\Lambda$ is a basic $\mathfrak{R}$-algebra, where $\mathfrak{R}$ is a complete local domain. Suppose that $T, U \in$ tilt $_{0} \Lambda$ are related by a mutation at an indecomposable summand $T_{n}$. If $T>U$, then there exists an exact sequence $0 \rightarrow \Lambda \rightarrow T^{\prime} \rightarrow T^{\prime \prime} \rightarrow 0$ such that $T_{n} \notin$ add $T^{\prime \prime}$.

Proof By the definition of tilting modules, there is an exact sequence $0 \rightarrow \Lambda \rightarrow T^{\prime} \rightarrow$ $T^{\prime \prime} \rightarrow 0$ with $T^{\prime}, T^{\prime \prime} \in$ add $T$, and this induces the following triangle in $\mathrm{D}^{\mathrm{b}}(\bmod \Lambda)$ :

$$
\Lambda \rightarrow T^{\prime} \rightarrow T^{\prime \prime} \stackrel{f}{\longrightarrow} \Lambda[1] .
$$

Since $\operatorname{Ext}^{1}(T, T)=0$, we see that $f: T^{\prime \prime} \rightarrow \Lambda[1]$ is a right add $T$-approximation. Replacing $T^{\prime}$ and $T^{\prime \prime}$ if necessary, we may assume that the approximation $f$ is rightminimal, and we will show that, for such a sequence $0 \rightarrow \Lambda \rightarrow T^{\prime} \rightarrow T^{\prime \prime} \rightarrow 0$, we have $T_{n} \notin$ add $T^{\prime \prime}$.

Suppose that $T_{n} \in$ add $T^{\prime \prime}$, and let $Y$ be the summand of $T^{\prime \prime}$ such that $T_{n} \notin$ add $Y$ and $T^{\prime \prime}=\left(T_{n}\right)^{\oplus a} \oplus Y$ for some $a>0$. Let $f_{n}:\left(T_{n}\right)^{\oplus a} \rightarrow \Lambda[1]$ and $f_{Y}: Y \rightarrow \Lambda[1]$ be the components of $f$. By assumption, there are indecomposable modules $T_{n}$ and $U_{n}$ such that $T=X \oplus T_{n}$ and $U=X \oplus U_{n}$. Since $T>U$, there is an exact sequence

$$
0 \rightarrow T_{n} \stackrel{g}{\longrightarrow} X^{\prime} \rightarrow U_{n} \rightarrow 0
$$

where $X^{\prime} \in \operatorname{add} X$. Applying $\operatorname{Hom}_{D^{b}(\bmod \Lambda)}(-, \Lambda[1])$ to the above gives an exact sequence

$\operatorname{Hom}_{\mathrm{D}^{\mathrm{b}}(\bmod \Lambda)}\left(X^{\prime}, \Lambda[1]\right) \stackrel{\circ g}{\longrightarrow} \operatorname{Hom}_{\mathrm{D}^{\mathrm{b}}(\bmod \Lambda)}\left(T_{n}, \Lambda[1]\right)$

$$
\rightarrow \operatorname{Hom}_{\mathrm{D}^{\mathrm{b}}(\bmod \Lambda)}\left(U_{n}[-1], \Lambda[1]\right)=0,
$$

since $\operatorname{pd}_{\Lambda} U_{n} \leq 1$. Hence, there exists a morphism $h: X^{\prime \oplus a} \rightarrow \Lambda[1]$ such that $f_{n}=$ $h \circ g^{\oplus a}$. But $h+f_{Y}: X^{\prime \oplus a} \oplus Y \rightarrow \Lambda[1]$ is a right add $T$-approximation, with $X^{\prime \oplus a} \oplus Y \in \operatorname{add} X$, and so $T_{n} \notin \operatorname{add}\left(X^{\prime \oplus a} \oplus Y\right)$. This contradicts the minimality of $f: T^{\prime \prime} \rightarrow \Lambda[1]$, since $T_{n} \in$ add $T^{\prime \prime}$. Hence, $T_{n} \notin$ add $T^{\prime \prime}$.

Theorem B.4 (Theorem 3.5) Suppose that $\Lambda$ is a basic $\mathfrak{R}$-algebra, where $\mathfrak{R}$ is a complete local domain. Suppose that $T, U \in$ tilt $_{0} \Lambda$ are related by a mutation at an indecomposable summand, so, by Lemma B.2, $C_{T}$ and $C_{U}$ are separated by $H$. Suppose that $[\Lambda] \notin H$. Then $T>U$ if and only if $C_{T}$ lies on the same side of $H$ as $[\Lambda]$. 
Proof $(\Longrightarrow)$ Suppose that $T>U$. Since the summands of $T$ (excluding $T_{0}=P_{0}$ ) form a basis for $\Theta_{\Lambda}$, we can write

$$
[\Lambda]=b_{1}\left[T_{1}\right]+\cdots+b_{n-1}\left[T_{n-1}\right]+b_{n}\left[T_{n}\right] .
$$

Certainly $b_{n} \neq 0$, otherwise $[\Lambda] \in H$, which is false by assumption. Since by Lemma B.3 there are objects $T^{\prime}, T^{\prime \prime} \in$ add $T$ such that $[\Lambda]=\left[T^{\prime}\right]-\left[T^{\prime \prime}\right]$ and $T_{n} \notin$ add $T^{\prime \prime}$, necessarily $b_{n}>0$ given that it is nonzero. It follows that $[\Lambda]$ is on the same side of $H$ as $C_{T}$.

( $\Longleftarrow$ ) If $\neg(T>U)$, then since by the assumption $T$ and $U$ are the mutation of each other at an indecomposable summand, necessarily $U>T$. Replicating the above proof word-for-word, we conclude that $C_{U}$ is on the same side of $H$ as $[\Lambda]$. Since $C_{T}$ is on the other side of $H$ than $C_{U}$, it follows that $C_{T}$ is not on the same side of $H$ as $[\Lambda]$.

\section{References}

[1] M Van den Bergh, Three-dimensional flops and noncommutative rings, Duke Math. J. 122 (2004) 423-455 MR

[2] A Bondal, D Orlov, Reconstruction of a variety from the derived category and groups of autoequivalences, Compositio Math. 125 (2001) 327-344 MR

[3] C Brav, H Thomas, Braid groups and Kleinian singularities, Math. Ann. 351 (2011) 1005-1017 MR

[4] S Brenner, M C R Butler, A spectral sequence analysis of classical tilting functors, from "Handbook of tilting theory" (L Angeleri Hügel, D Happel, H Krause, editors), London Math. Soc. Lecture Note Ser. 332, Cambridge Univ. Press (2007) 31-48 MR

[5] H Cartan, S Eilenberg, Homological algebra, Princeton Univ. Press (1956)

[6] I Cheltsov, C Shramov, Cremona groups and the icosahedron, CRC, Boca Raton, FL (2016) MR

[7] P Deligne, Les immeubles des groupes de tresses généralisés, Invent. Math. 17 (1972) 273-302 MR

[8] L Demonet, O Iyama, G Jasso, $\tau$-tilting finite algebras, bricks and g-vectors, Int. Math. Res. Not. (online publication July 2017) art. id. rnx135

[9] P Deshpande, Arrangements of submanifolds and the tangent bundle complement, $\mathrm{PhD}$ thesis, The University of Western Ontario (2011) Available at http:// ir.lib.uwo.ca/etd/154

[10] W Donovan, M Wemyss, Twists and braids for general 3-fold flops, preprint (2015) arXiv 
[11] W Donovan, M Wemyss, Noncommutative deformations and flops, Duke Math. J. 165 (2016) 1397-1474 MR

[12] D Happel, I Reiten, S O Smalø, Tilting in abelian categories and quasitilted algebras, Mem. Amer. Math. Soc. 575, Amer. Math. Soc., Providence, RI (1996) MR

[13] D Happel, L Unger, On a partial order of tilting modules, Algebr. Represent. Theory 8 (2005) 147-156 MR

[14] L Hille, On the volume of a tilting module, Abh. Math. Sem. Univ. Hamburg 76 (2006) 261-277 MR

[15] O Iyama, I Reiten, Fomin-Zelevinsky mutation and tilting modules over Calabi-Yau algebras, Amer. J. Math. 130 (2008) 1087-1149 MR

[16] O Iyama, M Wemyss, Affine actions on 3-folds via contracted preprojective algebras and Tits cone intersections, in preparation

[17] O Iyama, M Wemyss, Maximal modifications and Auslander-Reiten duality for nonisolated singularities, Invent. Math. 197 (2014) 521-586 MR

[18] O Iyama, M Wemyss, Reduction of triangulated categories and maximal modification algebras for $c A_{n}$ singularities, J. Reine Angew. Math. (online publication September 2015)

[19] S Katz, Small resolutions of Gorenstein threefold singularities, from "Algebraic geometry" (B Harbourne, R Speiser, editors), Contemp. Math. 116, Amer. Math. Soc., Providence, RI (1991) 61-70 MR

[20] L Paris, The covers of a complexified real arrangement of hyperplanes and their fundamental groups, Topology Appl. 53 (1993) 75-103 MR

[21] L Paris, Universal cover of Salvetti's complex and topology of simplicial arrangements of hyperplanes, Trans. Amer. Math. Soc. 340 (1993) 149-178 MR

[22] L Paris, On the fundamental group of the complement of a complex hyperplane arrangement, from "Arrangements" (M Falk, H Terao, editors), Adv. Stud. Pure Math. 27, Kinokuniya, Tokyo (2000) 257-272 MR

[23] H C Pinkham, Factorization of birational maps in dimension 3, from "Singularities, II” (P Orlik, editor), Proc. Sympos. Pure Math. 40, Amer. Math. Soc., Providence, RI (1983) 343-371 MR

[24] C Riedtmann, A Schofield, On a simplicial complex associated with tilting modules, Comment. Math. Helv. 66 (1991) 70-78 MR

[25] M Salvetti, Topology of the complement of real hyperplanes in $\mathbf{C}^{N}$, Invent. Math. 88 (1987) 603-618 MR

[26] P Seidel, R Thomas, Braid group actions on derived categories of coherent sheaves, Duke Math. J. 108 (2001) 37-108 MR 
[27] Y Sekiya, K Yamaura, Tilting theoretical approach to moduli spaces over preprojective algebras, Algebr. Represent. Theory 16 (2013) 1733-1786 MR

[28] R G Swan, Induced representations and projective modules, Ann. of Math. 71 (1960) 552-578 MR

[29] M Wemyss, Flops and clusters in the homological minimal model programme, Invent. Math. 211 (2018) 435-521 MR

Department of Mathematics, Kyoto University

Kyoto, Japan

School of Mathematics and Statistics, University of Glasgow

Glasgow, United Kingdom

y.hirano@math.kyoto-u.ac.jp, michael.wemyss@glasgow.ac.uk

Proposed: Richard Thomas

Seconded: Jim Bryan, Dan Abramovich
Received: 13 January 2017

Revised: 14 November 2017 
